\title{
Correlation of the highest-energy cosmic rays with the positions of nearby active galactic nuclei
}

\section{The Pierre Auger Collaboration}

J. Abraham ${ }^{\text {n }}$, P. Abreu ${ }^{\text {bm }}$, M. Aglietta ${ }^{\text {ay }}$, C. Aguirre ${ }^{\mathrm{q}}$, D. Allard ${ }^{\text {af }}$, I. Allekotte ${ }^{\mathrm{g}}$,

J. Allen $^{\text {ce }}$, P. Allison ${ }^{\mathrm{cg}}$, J. Alvarez-Muñiz ${ }^{\mathrm{bt}}, \mathrm{M}$. Ambrosio ${ }^{\mathrm{bb}}$, L. Anchordoqui ${ }^{\mathrm{ct}, \mathrm{cf}}{ }^{\prime}$,

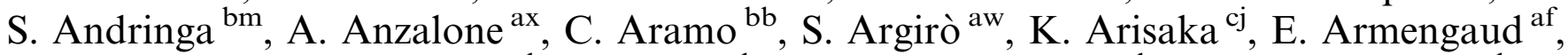
F. Arneodo ${ }^{\text {az }}$, F. Arqueros ${ }^{\text {bq }}$, T. Asch ${ }^{\text {al }}$, H. Asorey ${ }^{\mathrm{e}}$, P. Assis ${ }^{\mathrm{bm}}$, B.S. Atulugama ${ }^{\text {ch }}$, J. Aublin ${ }^{\text {ah }}$, M. Ave ${ }^{\text {ck }}$, G. Avila ${ }^{\mathrm{m}}$, T. Bäcker ${ }^{\text {ap }}$, D. Badagnani ${ }^{j}$, A.F. Barbosa ${ }^{\mathrm{s}}$, D. Barnhill ${ }^{\text {cj }}$, S.L.C. Barroso ${ }^{\mathrm{x}}$, P. Bauleo ${ }^{\mathrm{bz}}$, J.J. Beatty ${ }^{\mathrm{cg}}$, T. Beau ${ }^{\mathrm{af}}$, B.R. Becker ${ }^{\mathrm{cp}}$, K.H. Becker ${ }^{\text {aj }}$, J.A. Bellido ${ }^{\text {ch }}$, S. BenZvi ${ }^{\text {cs }}$, C. Berat ${ }^{\text {ai }}$, T. Bergmann ${ }^{\text {ao }}$, P. Bernardini ${ }^{\text {as }}$, X. Bertou ${ }^{\mathrm{e}}$, P.L. Biermann ${ }^{\text {am }}$, P. Billoir ${ }^{\text {ah }}$, O. Blanch-Bigas ${ }^{\text {ah }}$, F. Blanco ${ }^{\text {bq }}$, P. Blasi ${ }^{\text {cb,aq,ba }}$, C. Bleve ${ }^{\text {bv }}$, H. Blümer ${ }^{\text {ao,ak }}$, M. Boháčová ${ }^{\text {ad }}$, C. Bonifazi ${ }^{\text {ah,s }}$, R. Bonino ${ }^{\text {ay }}$, J. Brack $^{\text {bz,cl }}$, P. Brogueira ${ }^{\text {bm }}$, W.C. Brown ${ }^{\text {ca }}$, P. Buchholz ${ }^{\text {ap }}$, A. Bueno ${ }^{\text {bs }}$, R.E. Burton ${ }^{\text {bx }}$, N.G. Busca ${ }^{\text {af }}$, K.S. Caballero-Mora ${ }^{\text {ao }}$, B. Cai ${ }^{\text {cn }}$, D.V. Camin ${ }^{\text {ar }}$, L. Caramete ${ }^{\text {am }}$, R. Caruso ${ }^{\text {av }}$, W. Carvalho ${ }^{\text {u }}$, A. Castellina ay, O. Catalano ${ }^{\text {ax }}$, G. Cataldi ${ }^{\text {as }}$, L. Cazon $^{\text {ck }}$, R. Cester ${ }^{\text {aw }}$, J. Chauvin ${ }^{\text {ai }}$, A. Chiavassa ${ }^{\text {ay }}$, J.A. Chinellato ${ }^{v}$, A. Chou ${ }^{\text {ce,cb }}$, J. Chye ${ }^{\text {cd }}$, R.W. Clay ${ }^{\text {p }}$, E. Colombo ${ }^{\text {b }}$, R. Conceição ${ }^{\text {bm }}$, B. Connolly ${ }^{\mathrm{cq}}$, F. Contreras ${ }^{1}$, J. Coppens ${ }^{\text {bg,bi }}$, A. Cordier ${ }^{\text {ag }}$, U. Cotti ${ }^{\text {be }}$, S. Coutu ${ }^{\text {ch }}$, C.E. Covault ${ }^{\text {bx }}$, A. Creusot ${ }^{\text {bo }}$, A. Criss ${ }^{\text {ch }}$, J. Cronin ${ }^{\text {ck }}$, A. Curutiu ${ }^{\text {am }}$, S. Dagoret-Campagne ${ }^{\mathrm{ag}}$, K. Daumiller ${ }^{\mathrm{ak}}$, B.R. Dawson ${ }^{\mathrm{p}}$, R.M. de Almeida ${ }^{\mathrm{v}}$,

C. De Donato ${ }^{\text {ar }}$, S.J. de Jong ${ }^{\text {bg }}$, G. De La Vega ${ }^{\circ}$, W.J.M. de Mello Junior ${ }^{\mathrm{v}}$, J.R.T. de Mello Neto ${ }^{\text {ck,aa }}$, I. De Mitri ${ }^{\text {as }}$, V. de Souza ${ }^{\text {ao }}$, L. del Peral ${ }^{\text {br }}$, O. Deligny ${ }^{\text {ae }}$,

A. Della Selva ${ }^{\text {at }}$, C. Delle Fratte ${ }^{\text {au }}$, H. Dembinski ${ }^{\text {an }}$, C. Di Giulio ${ }^{\text {au }}$, J.C. Diaz ${ }^{\text {cd }}$, P.N. Diep ${ }^{\text {cu }}$, C. Dobrigkeit ${ }^{\mathrm{v}}$, J.C. D’Olivo ${ }^{\text {bf }}$, P.N. Dong ${ }^{\mathrm{cu}}$, D. Dornic ${ }^{\mathrm{ae}}$, A. Dorofeev $^{\mathrm{ce}}$, J.C. dos Anjos ${ }^{\mathrm{s}}$, M.T. Dova ${ }^{\mathrm{j}}$, D. D’Urso ${ }^{\text {at }}$, I. Dutan ${ }^{\mathrm{am}}$, M.A. DuVernois ${ }^{\mathrm{cm}}$, R. Engel ${ }^{\mathrm{ak}}$, L. Epele ${ }^{\mathrm{j}}$, M. Erdmann ${ }^{\text {an }}$, C.O. Escobar ${ }^{\mathrm{v}}$, A. Etchegoyen ${ }^{\mathrm{c}}$, P. Facal San Luis ${ }^{\mathrm{bt}}$, H. Falcke ${ }^{\text {bg,bj }}$, G. Farrar ${ }^{\text {ce }}$, A.C. Fauth ${ }^{\mathrm{v}}$, N. Fazzini ${ }^{\text {cb }}$, F. Ferrer ${ }^{\text {bx }}$, S. Ferry ${ }^{\text {bo }}$, B. Fick ${ }^{\text {cd }}$, A. Filevich ${ }^{\text {b }}$, A. Filipčič ${ }^{\text {bn,bo }}$, I. Fleck ${ }^{\text {ap }}$, C.E. Fracchiolla ${ }^{\mathrm{t}}$, W. Fulgione ${ }^{\text {ay }}$, B. García ${ }^{\mathrm{n}}$, D. García Gámez ${ }^{\text {bs }}$, D. Garcia-Pinto ${ }^{\text {bq }}$, X. Garrido ${ }^{\text {ag }}$, H. Geenen ${ }^{\text {aj }}$, G. Gelmini ${ }^{\text {cj }}$, H. Gemmeke ${ }^{\text {al }}$, P.L. Ghia ${ }^{\text {ae,ay }}$, M. Giller ${ }^{\text {bl }}$, H. Glass ${ }^{\text {cb }}$, M.S. Gold ${ }^{\text {cp }}$, G. Golup ${ }^{f}$, F. Gomez Albarracin ${ }^{j}$, M. Gómez Berisso ${ }^{\mathrm{f}}$, R. Gómez Herrero ${ }^{\text {br }}$, P. Gonçalves ${ }^{\mathrm{bm}}$, M. Gonçalves do Amaral ${ }^{\mathrm{ab}}$, D. Gonzalez ${ }^{\mathrm{ao}}$, J.G. Gonzalez ${ }^{\mathrm{cc}}, \mathrm{M}$. González ${ }^{\text {bd }}$, D. Góra ${ }^{\text {ao,bk }}$, A. Gorgi ${ }^{\text {ay }}$, P. Gouffon ${ }^{\text {u }}$, V. Grassi ${ }^{\text {ar }}$, A.F. Grillo ${ }^{\text {az }}$, C. Grunfeld ${ }^{j}$, Y. Guardincerri ${ }^{\text {h }}$, F. Guarino ${ }^{\text {at }}$, G.P. Guedes ${ }^{\text {w }}$, J. Gutiérrez ${ }^{\text {br }}$, J.D. Hague ${ }^{\text {cp }}$, J.C. Hamilton ${ }^{\text {af }}$, P. Hansen ${ }^{\text {bt }}$, D. Harari ${ }^{f}$, S. Harmsma ${ }^{\text {bh }}$, J.L. Harton ${ }^{\text {ae,bz }}$, A. Haungs ${ }^{\text {ak }}$, T. Hauschildt ${ }^{\text {ay }}$, M.D. Healy ${ }^{\text {cj }}$, T. Hebbeker $^{\text {an }}$, G. Hebrero ${ }^{\text {br }}$, D. Heck ${ }^{\text {ak }}$, C. Hojvat ${ }^{\text {cb }}$, V.C. Holmes ${ }^{p}$, P. Homola ${ }^{\text {bk }}$, J. Hörandel ${ }^{\text {bg }}$, A. Horneffer ${ }^{\text {bd }}$, M. Horvat ${ }^{\text {bo }}$, M. Hrabovský ${ }^{\text {ad }}$, T. Huege ${ }^{\text {ak }}$, M. Hussain ${ }^{\text {bo }}$, 
M. Iarlori ${ }^{\text {aq }}$, A. Insolia ${ }^{\text {av }}$, F. Ionita ${ }^{\text {ck }}$, A. Italiano ${ }^{\text {av }}$, M. Kaducak ${ }^{\text {cb }}$, K.H. Kampert ${ }^{\text {aj }}$, T. Karova ${ }^{\text {ad }}$, B. Kégl ${ }^{\text {ag }}$, B. Keilhauer ${ }^{\text {ao }}$, E. Kemp ${ }^{\text {v }}$, R.M. Kieckhafer ${ }^{\text {cd }}$, H.O. Klages ${ }^{\text {ak }}$, M. Kleifges ${ }^{\text {al }}$, J. Kleinfeller ${ }^{\text {ak }}$, R. Knapik ${ }^{\text {bz }}$, J. Knapp ${ }^{\text {bv }}$, D.-H. Koang ${ }^{\text {ai }}$, A. Krieger ${ }^{\text {b }}$, O. Krömer ${ }^{\text {al }}$, D. Kuempel ${ }^{\text {aj }}$, N. Kunka ${ }^{\text {al }}$, A. Kusenko ${ }^{\text {cj }}$, G. La Rosa ${ }^{\text {ax }}$, C. Lachaud ${ }^{\text {af }}$, B.L. Lago ${ }^{\text {aa }}$, D. Lebrun ${ }^{\text {ai }}$, P. LeBrun ${ }^{\text {cb }}$, J. Lee ${ }^{\text {cj }}$, M.A. Leigui de Oliveira ${ }^{z}$, A. Letessier-Selvon ${ }^{\text {ah }}$, M. Leuthold ${ }^{\text {an }}$, I. Lhenry-Yvon ${ }^{\text {ae }}$, R. López ${ }^{\text {bc }}$, A. Lopez Agüera ${ }^{\text {bt }}$, J. Lozano Bahilo ${ }^{\text {bs }}$, R. Luna García bd, M.C. Maccarone ${ }^{\text {ax }}$, C. Macolino ${ }^{\text {aq }}$, S. Maldera ${ }^{\text {ay }}$, G. Mancarella $^{\text {as }}$, M.E. Manceñido ${ }^{j}$, D. Mandat ${ }^{\mathrm{ad}}$, P. Mantsch $^{\mathrm{cb}}{ }^{\text {, A.G. Mariazzi }}{ }^{\mathrm{j}}$, I.C. Maris $^{\text {ao }}$, H.R. Marquez Falcon ${ }^{\text {be }}$, D. Martello ${ }^{\text {as }}$, J. Martínez ${ }^{\text {bd }}$, O. Martínez Bravo ${ }^{\text {bc }}$, H.J. Mathes ${ }^{\text {ak }}$, J. Matthews ${ }^{\text {cc,ci }}$, J.A.J. Matthews ${ }^{\text {cp }}$, G. Matthiae ${ }^{\text {au }}$, D. Maurizio ${ }^{\text {aw }}$, P.O. Mazur ${ }^{\mathrm{cb}}$, T. McCauley ${ }^{\mathrm{cf}}$, M. McEwen ${ }^{\text {br }}$, R.R. McNeil ${ }^{\mathrm{cc}}$, M.C. Medina ${ }^{\mathrm{c}}$, G. Medina-Tanco ${ }^{\text {bf }}$, A. Meli ${ }^{a m}$, D. Melo ${ }^{\text {b }}$, E. Menichetti ${ }^{\text {aw }}$, A. Menschikov ${ }^{\text {al }}$, Chr. Meurer ${ }^{\text {ak }}$, R. Meyhandan ${ }^{\text {bh }}$, M.I. Micheletti ${ }^{\text {c }}$, G. Miele ${ }^{\text {at }}$, W. Miller ${ }^{\text {cp }}$, S. Mollerach ${ }^{\text {f }}$, M. Monasor ${ }^{\text {bq,br }}$, D. Monnier Ragaigne ${ }^{\text {ag }}$, F. Montanet ${ }^{\text {ai }}$, B. Morales ${ }^{\text {bf }}$, C. Morello ${ }^{\text {ay }}$, J.C. Moreno ${ }^{j}$, C. Morris ${ }^{\text {cg }}$, M. Mostafá ${ }^{\text {cr }}$, M.A. Muller ${ }^{\mathrm{v}}$, R. Mussa ${ }^{\text {aw }}$, G. Navarra ${ }^{\text {ay }}$, J.L. Navarro ${ }^{\text {bs }}$, S. Navas ${ }^{\text {bs }}$, P. Necesal ${ }^{\text {ad }}$, L. Nellen ${ }^{\text {bf }}$, C. Newman-Holmes ${ }^{\text {cb }}$, D. Newton ${ }^{\text {bv,bt }}$, P.T. Nhung ${ }^{\text {cu }}$, N. Nierstenhoefer ${ }^{\text {aj }}$, D. Nitz ${ }^{\text {cd }}$, D. Nosek ${ }^{\text {ac }}$, L. Nožka ${ }^{\text {ad }}$, J. Oehlschläger $^{\text {ak }}$,

T. Ohnuki ${ }^{\text {cj }}$, A. Olinto ${ }^{\text {af,ck }}$, V.M. Olmos-Gilbaja ${ }^{\text {bt }}$, M. Ortiz ${ }^{\text {bq }}$, F. Ortolani ${ }^{\text {au }}$,

S. Ostapchenko ${ }^{\text {ao }}$, L. Otero $^{\text {n }}$, N. Pacheco ${ }^{\text {br }}$, D. Pakk Selmi-Dei ${ }^{\text {v }}$, M. Palatka ${ }^{\text {ad }}$, J. Pallotta ${ }^{\text {a }}$, G. Parente ${ }^{\text {bt }}$, E. Parizot ${ }^{\text {af }}$, S. Parlati ${ }^{\text {az }}$, S. Pastor ${ }^{\text {bp }}$, M. Patel ${ }^{\text {bv }}$, T. Paul $^{\text {cf }}$, V. Pavlidou ${ }^{\text {ck }}$, K. Payet ${ }^{\text {ai }}$, M. Pech ${ }^{\text {ad }}$, J. Pȩkala ${ }^{\text {bk }}$, R. Pelayo ${ }^{\text {bd }}$, I.M. Pepe ${ }^{y}$, L. Perrone ${ }^{\text {as }}$, S. Petrera ${ }^{\text {aq }}$, P. Petrinca ${ }^{\text {au }}$, Y. Petrov ${ }^{\text {bz }}$, A. Pichel $^{\mathrm{k}}$, R. Piegaia ${ }^{\mathrm{h}}$, T. Pierog $^{\text {ak }}$, M. Pimenta ${ }^{\mathrm{bm}}$, T. Pinto $^{\mathrm{bp}}$,

V. Pirronello $^{\text {av }}$, O. Pisanti ${ }^{\text {at }}$, M. Platino ${ }^{\mathrm{b}}$, J. Pochon ${ }^{\mathrm{e}}$, P. Privitera ${ }^{\text {au }}$, M. Prouza ${ }^{\text {ad }}$, E.J. Quel ${ }^{\mathrm{a}}$, J. Rautenberg ${ }^{\mathrm{aj}}$, A. Redondo ${ }^{\mathrm{br}}$, S. Reucroft ${ }^{\mathrm{cf}}$, B. Revenu ${ }^{\text {af }}$, F.A.S. Rezende ${ }^{\mathrm{s}}$, J. Ridky ${ }^{\text {ad }}$, S. Riggi ${ }^{\text {av }}$, M. Risse ${ }^{\text {aj }}$, C. Rivière ${ }^{\text {ai }}$, V. Rizi ${ }^{\text {aq }}$, M. Roberts ${ }^{\text {ch }}$, C. Robledo ${ }^{\text {bc }}$, G. Rodriguez $^{\mathrm{bt}}$, J. Rodriguez Martino ${ }^{\mathrm{av}}$, J. Rodriguez Rojo ${ }^{1}$, I. Rodriguez-Cabo ${ }^{\mathrm{bt}}$, M.D. Rodríguez-Frías ${ }^{\text {br }}$, G. Ros ${ }^{\text {bq,br }}{ }^{\text {J. J. Rosado }}{ }^{\text {bq }}$, M. Roth ${ }^{\text {ak }}$, B. Rouillé-d'Orfeuil af, E. Roulet ${ }^{\mathrm{f}}$, A.C. Rovero ${ }^{\mathrm{k}}$, F. Salamida ${ }^{\mathrm{aq}}$, H. Salazar ${ }^{\mathrm{bc}}$, G. Salina ${ }^{\mathrm{au}}$, F. Sánchez ${ }^{\text {bf }}$, M. Santander ${ }^{1}$, C.E. Santo ${ }^{\text {bm }}$, E.M. Santos ${ }^{\text {ah,s }}$, F. Sarazin ${ }^{\text {by }}$, S. Sarkar ${ }^{\text {bu }}$, R. Sato ${ }^{1}$, V. Scherini ${ }^{\text {aj }}$, H. Schieler ${ }^{\text {ak }}$, A. Schmidt ${ }^{\text {al }}$, F. Schmidt ${ }^{\text {ck }}$, T. Schmidt ${ }^{\text {ao }}$, O. Scholten ${ }^{\text {bh }}$, P. Schovánek ${ }^{\text {ad }}$, F. Schüssler ${ }^{\text {ak }}$, S.J. Sciutto ${ }^{j}$, M. Scuderi ${ }^{\text {av }}$, A. Segreto ${ }^{\text {ax }}$, D. Semikoz ${ }^{\text {af }}$, M. Settimo ${ }^{\text {as }}$, R.C. Shellard ${ }^{\text {s,t }}$, I. Sidelnik $^{\text {c }}$, B.B. Siffert ${ }^{\text {aa }}$, G. Sigl ${ }^{\text {af }}$,

N. Smetniansky De Grande ${ }^{\mathrm{b}}$, A. Smiałkowski ${ }^{\text {bl }}$, R. Šmída ${ }^{\text {ad }}$, A.G.K. Smith ${ }^{\mathrm{p}}$, B.E. Smith ${ }^{\text {bv }}$, G.R. Snow ${ }^{\text {co }}$, P. Sokolsky ${ }^{\text {cr }}$, P. Sommers ${ }^{\text {ch }}$, J. Sorokin ${ }^{p}$, H. Spinka ${ }^{\text {bw,cb }}$, R. Squartini ${ }^{1}$,

E. Strazzeri ${ }^{\text {au }}$, A. Stutz ${ }^{\text {ai }}$, F. Suarez ${ }^{\text {ay }}$, T. Suomijärvi ${ }^{\text {ae }}$, A.D. Supanitsky ${ }^{\text {bf }}$,

M.S. Sutherland ${ }^{\text {cg }}$, J. Swain ${ }^{\text {cf }}$, Z. Szadkowski ${ }^{\text {bl }}$, J. Takahashi ${ }^{\text {v }}$, A. Tamashiro ${ }^{k}$, A. Tamburro $^{\text {ao }}$, O. Taşcău ${ }^{\text {aj }}$, R. Tcaciuc ${ }^{\text {ap }}$, N.T. Thao ${ }^{\text {cu }}$, D. Thomas ${ }^{\text {cr }}$, R. Ticona ${ }^{r}$, J. Tiffenberg ${ }^{\mathrm{h}}$, C. Timmermans ${ }^{\text {bi,bg }}$, W. Tkaczyk ${ }^{\text {bl }}$, C.J. Todero Peixoto ${ }^{\mathrm{v}}$, B. Tomé ${ }^{\mathrm{bm}}$, A. Tonachini ${ }^{\text {aw }}$, I. Torres ${ }^{\text {bc }}$, P. Travnicek ${ }^{\text {ad }}$, A. Tripathi ${ }^{\text {cj }}$, G. Tristram ${ }^{\text {af }}$, D. Tscherniakhovski ${ }^{\text {al }}$, M. Tueros ${ }^{\mathrm{i}}$, R. Ulrich ${ }^{\mathrm{ak}}$, M. Unger $^{\text {ak }}, \mathrm{M}$. Urban ${ }^{\mathrm{ag}}$, J.F. Valdés Galicia ${ }^{\text {bf }}$, I. Valiño $^{\text {bt }}$, L. Valore $^{\text {at }}$, A.M. van den Berg ${ }^{\text {bh }}$, V. van Elewyck ${ }^{\text {ae }}$,

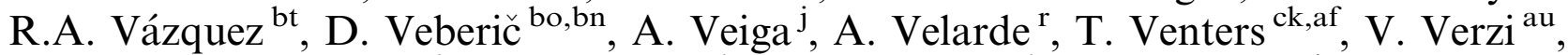
M. Videla ${ }^{\mathrm{a}}$, L. Villaseñor ${ }^{\text {be }}, \mathrm{S}$. Vorobiov ${ }^{\text {bo }}$, L. Voyvodic $^{\mathrm{cb}}, \mathrm{H}$. Wahlberg ${ }^{\mathrm{j}}$, O. Wainberg ${ }^{\mathrm{d}}$, D. Warner ${ }^{\text {bz }}$, A.A. Watson ${ }^{\text {bv }}$, S. Westerhoff ${ }^{\text {cs }}$, G. Wieczorek $^{\text {bl }}$, L. Wiencke ${ }^{\text {by }}$, 


\section{B. Wilczyńska ${ }^{\text {bk }}$, H. Wilczyński ${ }^{\text {bk }}$, C. Wileman ${ }^{\text {bv }}$, M.G. Winnick ${ }^{\mathrm{p}}, \mathrm{H} \mathrm{Wu}^{\text {ag }}$, B. Wundheiler ${ }^{\text {b }}$, T. Yamamoto ${ }^{\text {ck }}$, P. Younk ${ }^{\text {cr }}$, E. Zas ${ }^{\text {bt }}$, D. Zavrtanik ${ }^{\text {bo,bn }}$, M. Zavrtanik ${ }^{\text {bn,bo }}$, A. Zech ${ }^{\text {ah }}$, A. Zepeda ${ }^{\text {bd }}$, M. Ziolkowski ${ }^{\text {ap }}$}

${ }^{a}$ Centro de Investigaciones en Láseres y Aplicaciones, CITEFA and CONICET, Argentina

${ }^{\mathrm{b}}$ Centro Atómico Constituyentes, CNEA, Buenos Aires, Argentina

${ }^{\mathrm{c}}$ Centro Atómico Constituyentes, Comisión Nacional de Energía Atómica and CONICET, Argentina

${ }^{\mathrm{d}}$ Centro Atómico Constituyentes, Comisión Nacional de Energía Atómica and UTN-FRBA, Argentina

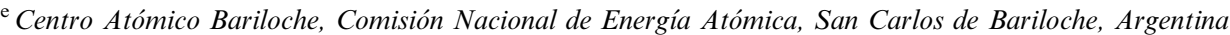

${ }^{\mathrm{f}}$ Departamento de Física, Centro Atómico Bariloche, Comisión Nacional de Energía Atómica and CONICET, Argentina

${ }^{\mathrm{g}}$ Centro Atómico Bariloche, Comision Nacional de Energía Atómica and Instituto Balseiro, CNEA-UNC, San Carlos de Bariloche, Argentina

${ }^{\mathrm{h}}$ Departamento de Física, FCEyN, Universidad de Buenos Aires y CONICET, Argentina

${ }^{i}$ Departamento de Física, Universidad Nacional de La Plata and Fundación Universidad Tecnológica Nacional, Argentina

${ }^{\mathrm{j}}$ IFLP, Universidad Nacional de La Plata and CONICET, La Plata, Argentina

${ }^{\mathrm{k}}$ Instituto de Astronomía y Física del Espacio, CONICET, Buenos Aires, Argentina

${ }^{1}$ Pierre Auger Southern Observatory, Malargüe, Argentina

${ }^{\mathrm{m}}$ Pierre Auger Southern Observatory and Comisión Nacional de Energía Atómica, Malargüe, Argentina

${ }^{\mathrm{n}}$ Universidad Tecnológica Nacional, FR-Mendoza, Argentina

${ }^{\circ}$ Universidad Tecnológica Nacional, FR-Mendoza and Fundación Universidad Tecnológica Nacional, Argentina

${ }^{\mathrm{p}}$ University of Adelaide, Adelaide, SA, Australia

${ }^{\mathrm{q}}$ Universidad Catolica de Bolivia, La Paz, Bolivia

${ }^{\mathrm{r}}$ Universidad Mayor de San Andrés, Bolivia

${ }^{\mathrm{s}}$ Centro Brasileiro de Pesquisas Fisicas, Rio de Janeiro, RJ, Brazil

${ }^{\mathrm{t}}$ Pontifícia Universidade Católica, Rio de Janeiro, RJ, Brazil

${ }^{u}$ Universidade de Sao Paulo, Instituto de Fisica, Sao Paulo, SP, Brazil

${ }^{\mathrm{v}}$ Universidade Estadual de Campinas, IFGW, Campinas, SP, Brazil

${ }^{\mathrm{w}}$ Universidade Estadual de Feira de Santana, Brazil

${ }^{\mathrm{x}}$ Universidade Estadual do Sudoeste da Bahia, Vitoria da Conquista, BA, Brazil

${ }^{\text {y } U n i v e r s i d a d e ~ F e d e r a l ~ d a ~ B a h i a, ~ S a l v a d o r, ~ B A, ~ B r a z i l ~}$

${ }^{\mathrm{z}}$ Universidade Federal do ABC, Santo André, SP, Brazil

${ }^{\text {aa }}$ Universidade Federal do Rio de Janeiro, Instituto de Física, Rio de Janeiro, RJ, Brazil

${ }^{\mathrm{ab}}$ Universidade Federal Fluminense, Instituto de Fisica, Niterói, RJ, Brazil

${ }^{\mathrm{ac}}$ Charles University, Institute of Particle \& Nuclear Physics, Prague, Czech Republic

${ }^{\mathrm{ad}}$ Institute of Physics of the Academy of Sciences of the Czech Republic, Prague, Czech Republic

${ }^{\mathrm{ae}}$ Institut de Physique Nucléaire, Université Paris-Sud, IN2P3/CNRS, Orsay, France

${ }^{\text {af }}$ Laboratoire AstroParticule et Cosmologie, Université Paris 7, IN2P3/CNRS, Paris, France

${ }^{a g}$ Laboratoire de l'Accélérateur Linéaire, Université Paris-Sud, IN2P3/CNRS, Orsay, France

${ }^{a h}$ Laboratoire de Physique Nucléaire et de Hautes Energies, Universités Paris 6 \& 7, IN2 P3/CNRS, Paris Cedex 05, France

${ }^{\text {ai }}$ Laboratoire de Physique Subatomique et de Cosmologie, IN2P3/CNRS, Université Grenoble 1 et INPG, Grenoble, France

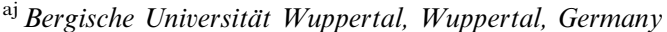

${ }^{\mathrm{ak}}$ Forschungszentrum Karlsruhe, Institut für Kernphysik, Karlsruhe, Germany

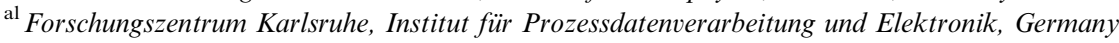

${ }^{\mathrm{am}}$ Max-Planck-Institut für Radioastronomie, Bonn, Germany

${ }^{a n}$ RWTH Aachen University, III. Physikalisches Institut A, Aachen, Germany

${ }^{\text {ao }}$ Universität Karlsruhe, TH, Institut für Experimentelle Kernphysik, IEKP, Karlsruhe, Germany

${ }^{\text {ap }}$ Universität Siegen, Siegen, Germany

${ }^{\text {aq }}$ Università de l'Aquila and Sezione INFN, Aquila, Italy

${ }^{\mathrm{ar}}$ Università di Milano and Sezione INFN, Milan, Italy

${ }^{\text {as }}$ Università del Salento and Sezione INFN, Lecce, Italy

at Università di Napoli "Federico II" and Sezione INFN, Napoli, Italy

au Università di Roma II "Tor Vergata" and Sezione INFN, Roma, Italy

${ }^{\text {av }}$ Università di Catania and Sezione INFN, Catania, Italy

${ }^{\text {aw }}$ Università di Torino and Sezione INFN, Torino, Italy

${ }^{\mathrm{ax}}$ Istituto di Astrofisica Spaziale e Fisica Cosmica di Palermo, INAF, Palermo, Italy

${ }^{\text {ay }}$ Istituto di Fisica dello Spazio Interplanetario, INAF, Università di Torino and Sezione INFN, Torino, Italy

${ }^{\mathrm{az}}$ INFN, Laboratori Nazionali del Gran Sasso, Assergi, L'Aquila, Italy

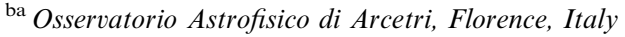

${ }^{\mathrm{bb}}$ Sezione INFN di Napoli, Napoli, Italy

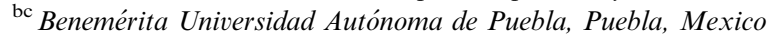

${ }^{\text {bd }}$ Centro de Investigación y de Estudios Avanzados del IPN, CINVESTAV, México, DF, Mexico

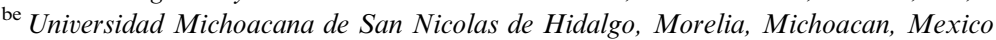

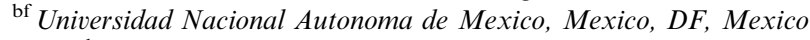

bg IMAPP, Radboud University, Nijmegen, Netherlands

${ }^{\text {bh }}$ Kernfysisch Versneller Instituut, University of Groningen, Groningen, Netherlands

${ }^{\text {bi }}$ NIKHEF, Amsterdam, Netherlands

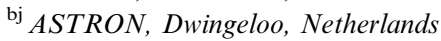




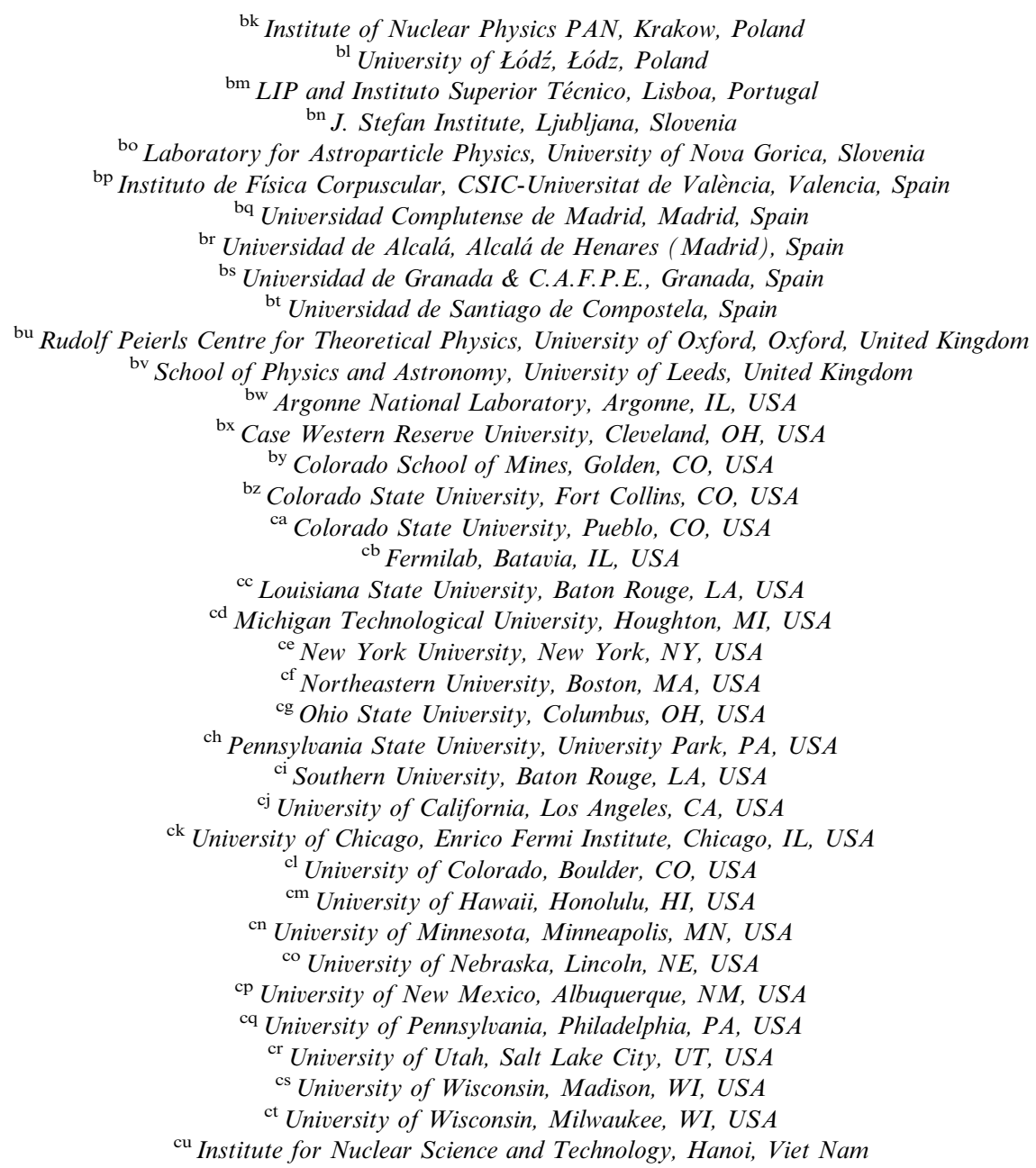

Received 15 December 2007; received in revised form 9 January 2008; accepted 9 January 2008 Available online 3 February 2008

\begin{abstract}
Data collected by the Pierre Auger Observatory provide evidence for anisotropy in the arrival directions of the cosmic rays with the highest-energies, which are correlated with the positions of relatively nearby active galactic nuclei (AGN) [Pierre Auger Collaboration, Science 318 (2007) 938]. The correlation has maximum significance for cosmic rays with energy greater than $\sim 6 \times 10^{19} \mathrm{eV}$ and AGN at a distance less than $\sim 75 \mathrm{Mpc}$. We have confirmed the anisotropy at a confidence level of more than $99 \%$ through a test with parameters specified a priori, using an independent data set. The observed correlation is compatible with the hypothesis that cosmic rays with the highest-energies originate from extra-galactic sources close enough so that their flux is not significantly attenuated by interaction with the cosmic background radiation (the Greisen-Zatsepin-Kuz'min effect). The angular scale of the correlation observed is a few degrees, which suggests a predominantly light composition unless the magnetic fields are very weak outside the thin disk of our galaxy. Our present data do not identify AGN as the sources of cosmic rays unambiguously, and other candidate sources which are distributed as nearby AGN are not ruled out. We discuss the prospect of unequivocal identification of individual sources of the highest-energy cosmic rays within a few years of continued operation of the Pierre Auger Observatory.
\end{abstract}

(C) 2008 Elsevier B.V. All rights reserved.

Keywords: High energy cosmic rays; UHECR; AGN; Anisotropy; Extra-galactic; Auger; Observatory; GZK; GMF; EGMF 


\section{Introduction}

The identification of the sources of the cosmic rays with the highest-energies so far detected has been a great challenge ever since the first event with energy around $10^{20} \mathrm{eV}$ was reported [2]. If the highest-energy cosmic rays are predominantly protons and nuclei, only sources which are less than about $200 \mathrm{Mpc}$ from earth could contribute significantly to the observed flux above $6 \times 10^{19} \mathrm{eV}$. Protons with higher energies interact with cosmic microwave background photons to produce pions [3,4], which leads to a significant attenuation of their flux from more distant sources. The energy of light nuclei is damped over an even shorter length scale due to photo-disintegration processes $[5,6]$. If the relatively nearby sources are not uniformly distributed then we expect that the arrival directions of the most energetic cosmic rays should be anisotropic, as long as deflections imprinted by intervening magnetic fields upon their trajectories are small enough that they point back to their place of origin.

The Pierre Auger Observatory [7], has been operating in Argentina and taking data in a stable mode since January 2004. The large exposure of the surface detectors (SD), combined with accurate energy and arrival direction measurements, calibrated and verified from the hybrid operation with fluorescence detectors (FD), provides an opportunity to find the clues that could lead to an understanding of the origin of the highest-energy cosmic rays.

AGN have long been considered possible sites for energetic particle production, where protons and heavier nuclei could be accelerated up to the highest-energies measured so far $[8,9]$. Windows of a few degrees around each known AGN lying within $100 \mathrm{Mpc}$ cover a significant fraction but not most - of the sky. We were therefore motivated to search for an excess, as compared to expectations for an isotropic flux, of cosmic rays with arrival directions close to AGN. The angular size of the search window should not be limited to the instrumental angular resolution, since correlation could exist on larger scales due to magnetic deflections, the precise amount of which is unknown. Arrival directions of cosmic rays are reconstructed by the SD array

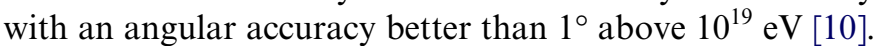

We have recently reported [1] the observation of a correlation between the arrival directions of the cosmic rays with highest-energies measured by the Pierre Auger Observatory and the positions of nearby AGN from the 12th edition of the catalogue of quasars and active nuclei by VéronCetty and Véron [11]. In this article we provide more details about the methods used to demonstrate anisotropy based on this correlation, and further analyse its properties and implications.

\section{Evidence for anisotropy and correlation with AGN}

\subsection{Data set}

The southern site of the Pierre Auger Observatory [7] is located in Malargüe, Argentina, at latitude $35.2^{\circ} \mathrm{S}$, longitude $69.5^{\circ} \mathrm{W}$, and mean altitude $1400 \mathrm{~m}$ above sea level. The data set analysed here consists of events recorded by the Pierre Auger Observatory from 1 January 2004 to 31 August 2007. During this time, the size of the Observatory increased from 154 to 1388 surface detector stations. We consider events with reconstructed energies above $40 \mathrm{EeV}$ $\left(1 \mathrm{EeV}=10^{18} \mathrm{eV}\right)$ and zenith angles smaller than $60^{\circ}$. The quality cut implemented in the present analysis requires that at least five active nearest neighbours surround the station with the highest signal when the event was recorded, and that the reconstructed shower core be inside an active equilateral triangle of detectors.

The event direction is determined by a fit of the arrival times of the shower front at the SD. The precision achieved in the arrival direction depends on the clock resolution of each detector and on the fluctuations in the time of arrival of the first particle [12]. The angular resolution is defined as the angular aperture around the arrival directions of cosmic rays within which $68 \%$ of the showers are reconstructed. This resolution has been verified experimentally $[13,10]$. Almost all events with energies above $10 \mathrm{EeV}$ trigger at least six surface stations and have an angular resolution better than $1^{\circ}[13,10]$.

The energy of each event is determined in a two-step procedure. The shower size $S$, at a reference distance and zenith angle, is calculated from the signal detected in each surface station and then converted to energy using a linear calibration curve based on the fluorescence telescope measurements [14]. The uncertainty in $S$ resulting from the adjustment of the shower size, the conversion to a reference angle, the fluctuations from shower-to-shower and the calibration curve amounts to about $18 \%$. The absolute energy scale is given by the fluorescence measurements and has a systematic uncertainty of $22 \%$ [15]. There is an additional uncertainty in the energy scale for the set of high energy events used in the present analysis due to the relatively low statistics available for calibration in this energy range.

\subsection{Exposure}

The integrated exposure for the event selection described in the previous section amounts to $9000 \mathrm{~km}^{2} \mathrm{sr} \mathrm{yr}$. Note that analyses involving a flux calculation, such as the estimate of the cosmic ray spectrum [14], use stricter selection criteria which would amount to an exposure of about $7000 \mathrm{~km}^{2} \mathrm{sr}$ yr for the same data period.

The surface detector array has full acceptance for events with energy above $3 \mathrm{EeV}$ [16]. Above this energy the detection efficiency is larger than $99 \%$ and it is nearly independent of the direction of the shower axis defined by the zenith angle $(\theta)$ with respect to the local vertical and azimuth $(\phi)$ with respect to the South. Thus, above that energy the instantaneous instrument aperture as a function of zenith angle is given by

$A(t)=n(t) a_{0} \cos \theta \mathrm{d} \Omega \mathrm{d} t$, 
where $a_{0} \cos \theta$ is the surface of a unitary cell under the incidence zenith angle $\theta$ and $n(t)$ is the number of active such cells as a function of time. The number $n(t)$ is recorded every second by the trigger system of the Observatory and reflects the array growth as well as the dead period of each detector. Such recording allows for a precise knowledge of our aperture at any moment in time.

The instrument exposure above a certain energy $E$ may be further affected by the conversion of the measured signal at ground to energy (this dependence is not included in Eq. (1) above). For a given energy $E$ the ground signal vary depending on the atmospheric conditions (e.g. through the variations of the Molière radius) [17]. If the signal to energy conversion does not correct for these small variations, of order a few $\%$, as it is the case in our analysis, the aperture above a certain uncorrected energy will depend on the atmospheric conditions.

Over the period from 1 January 2004 to 31 August 2007 the integration of the time dependences from the array growth and dead time together with the atmospheric variations introduce a modulation of the exposure as a function of celestial right ascension (RA) of less than $1 \%$. For the purpose of our analysis, where the total number of events considered is less than 100 , such modulation is negligible and the resulting RA dependence can be safely ignored.

Hence our exposure only depends on the celestial declination $\delta$ and can be derived from the relation $\sin \delta=\cos \theta \sin \lambda-\sin \theta \cos \lambda \cos \phi$, where $\lambda$ is the latitude of the Observatory.

\subsection{Search method}

We denote by $p$ the probability that an individual event from an isotropic flux has, by chance, an arrival direction closer than some particular angular distance $\psi$ from any member of a collection of candidate point sources. $p$ is the exposure-weighted fraction of the sky accessible to observation by the Pierre Auger Observatory which is covered by windows of radius $\psi$ centred on the selected sources.

The probability $P$ that $k$ or more out of a total of $N$ events from an isotropic flux are correlated by chance with the selected objects at the chosen angular scale is given by the cumulative binomial distribution

$P=\sum_{j=k}^{N}\left(\begin{array}{c}N \\ j\end{array}\right) p^{j}(1-p)^{N-j}$.

For this analysis we consider the correlation between cosmic rays and AGN in the 12th edition of the catalogue of quasars and active nuclei by Véron-Cetty and Véron [11] (V-C). This catalogue can not be claimed to contain all existing AGN, nor to be an unbiased statistical sample of them. It, however, contains the results of a thorough survey of all such objects in the literature. This catalogue contains 85,221 quasars, $1122 \mathrm{BL}$ Lac objects and 21,737 active galaxies. Among these objects, 694 have redshift $z \leqslant 0.024$, a value corresponding to a distance smaller than approximately $100 \mathrm{Mpc}{ }^{1}$ At distances greater than $100 \mathrm{Mpc}$ the catalogue becomes increasingly incomplete and inhomogeneous. The $\mathrm{V}-\mathrm{C}$ catalogue is also particularly incomplete around the galactic plane. This is not an obstacle to demonstrating the existence of anisotropy but may affect our ability to identify the cosmic ray sources unambiguously.

We compute the degree of correlation as a function of three parameters: the maximum AGN redshift $z_{\max }$, the maximum angular separation $\psi$, and the lower threshold energy for cosmic rays $E_{\text {th }}$. Our scan in angular separation $\psi$ is constrained by the angular resolution of the $\mathrm{SD}[10]$ at the low end (we use $\psi_{\min }=1^{\circ}$ ) and by the increase in the individual probability $p$ at the high end; large $\psi$ push the value of $p$ toward unity, rendering searches for correlation above isotropic expectations meaningless. Illustrative values are $p=0.27$ and 0.6 for maximum angular distance $\psi=3^{\circ}$ and $6^{\circ}$, respectively, in the case of maximum AGN redshift $z_{\max }=0.024$. Our scan in energies is motivated by the assumption that the highest-energy cosmic rays are those that are least deflected by intervening magnetic fields, and that they have a smaller probability to arrive from very distant sources due to the GZK effect $[3,4]$. The scan in energy threshold is carried out starting with the event with the highest-energy and adding, one by one, events with successively lower energy.

We scan with the method described above to find the minimum value of $P$, given in Eq. (2). Note, however, that $P_{\min }$ is not the chance probability that the observed arrival directions are isotropically distributed. An estimate of the chance probability must incorporate the effect of the scan performed upon the data. To do so, we build simulated sets, each having the same number of events as in the data set, drawn from an isotropic flux in proportion to the relative exposure of the Observatory. The chance probability is estimated from the fraction of simulated isotropic sets that have, anywhere in the parameter space and under the same scan, equal or smaller values of $P_{\min }$ than the minimum found in the data [18]. The result can only be considered an estimate of the chance probability, since it depends somewhat on the choice of the range for the scan parameters, and does not account for the possibility of dilution due to different scan methods or for scans against different sets of astronomical objects.

\subsection{Exploratory scan and anisotropy confirmation}

An exploratory search for correlation between cosmic rays and AGN was conducted according to the method described in Section 2.3 using data collected from 1 January 2004 to 27 May 2006. This search yielded a minimum

\footnotetext{
${ }^{1}$ For a redshift $z$ small compared to 1 , the distance to an object is approximately $42 \mathrm{Mpc} \times(z / 0.01)$ for a Hubble constant $H_{0}=$ $71 \mathrm{~km} \mathrm{~s}^{-1} \mathrm{Mpc}^{-1}$.
} 
probability $P_{\min }$ for the parameter set: $z_{\max }=0.018$ $\left(D_{\max }=75 \mathrm{Mpc}\right), \quad E_{\mathrm{th}}=56 \mathrm{EeV}$ and $\psi=3.1^{\circ}, \quad$ with 12 events among 15 correlated with at least one of the selected AGN. For this parameter set, the chance correlation is $p=0.21$. Only 3.2 events were expected to correlate by chance if the flux was isotropic.

Much of the discussion regarding past evidence for possible anisotropy in the distribution of the arrival directions of ultra-high energy cosmic rays has been centred on the issue of the impact of trial factors on the statistical significance of any potential signal. An accurate measure of the statistical significance of some previous reports of anisotropy [19-22], could not be achieved due to the posterior nature of the analyses involved. It is only from subsequent observations that those claims could be quantitatively evaluated [23-27].

Therefore, to avoid the negative impact of trial factors in a posteriori anisotropy searches, the Pierre Auger collaboration decided that any potentially interesting anisotropy signal should be tested on an independent data set with parameters specified a priori. This method was described in [28], where a particular set of parameters and sources were proposed and subsequently tested on the first Auger data set [29].

The correlation observed in the exploratory scan motivated the construction of a specific test to reject or accept the isotropy hypothesis with parameters specified a priori on an independent data set, using exactly the same reconstruction algorithms, energy calibration and quality cuts for event selection as in the exploratory scan. All details of the prescribed test were documented and archived in an internal note.

The test null hypothesis is isotropy and its statistical characteristics are fully defined by the choice of two probabilities known as the type I and type II errors. The type I error $(\alpha)$ is the probability of rejecting the null hypothesis incorrectly. In our case, this is the probability of declaring our independent data set anisotropic when it is not. We have chosen $\alpha=1 \%$. The type II error $(\beta)$ is the probability of accepting the null hypothesis incorrectly. In our case this is the probability of declaring the independent data set isotropic when it is not. We have chosen $\beta=5 \%$.

The selection and correlation criteria for the events were chosen according to the parameter set that minimised the probability in the exploratory scan $\left(\psi=3.1^{\circ}, z_{\max }=\right.$ $\left.0.018, E_{\text {th }}=56 \mathrm{EeV}\right)$. Since we could not predict how many events would be required to confirm the results at a statistically significant level from the exploratory scan, we adopted a running prescription (with a pre-defined stopping rule) for conducting a sequential analysis with individual tests to be applied after the detection of each subsequent event passing our selection criteria.

If, in the sequence, one of the individual tests is satisfied, we reject the hypothesis of isotropy with a confidence level of at least $(1-\alpha)=99 \%$. The total length of the test sequence ( 34 events) was determined by the requirement of detecting a minimum correlation power of $60 \%$, as esti-
Table 1

Criteria for our running prescription where $N$ corresponds to the total number of events observed at any point during the sequential analysis of up to 34 events arriving with energy $E>56 \mathrm{EeV}$

\begin{tabular}{lllllllllll}
\hline$N$ & 4 & 6 & 8 & 10 & 12 & $\ldots$ & 30 & 31 & 33 & 34 \\
$k_{\min }$ & 4 & 5 & 6 & 7 & 8 & $\ldots$ & 14 & 14 & 15 & 15 \\
\hline
\end{tabular}

$k_{\min }$ is the minimum number of events within the angular window $\left(\psi=3.1^{\circ}\right)$, and a maximum AGN redshift $\left(z_{\max }=0.018\right)$ required to reject isotropy with at least a $99 \%$ confidence level. This prescription applied to data collected after 27 May 2006 was satisfied with $N=8$ and $k=6$ on 25 May 2007.

mated from the statistics of the exploratory scan, within our specified $\beta$ of $5 \%$. In Table 1 we list, for a given number of events passing our selection criteria $N$, the minimum number of events in correlation $k_{\min }$ necessary to reject the null hypothesis (isotropy) with a confidence level larger than $99 \%$, accounting for the sequential nature and finite length of the test. Note that for some values of $N$ (e.g. 5, 7,11 , etc.) there exists no value of $k_{\min }$ that can satisfy the threshold probability without also having already satisfied the threshold at a lower value of $N$.

The prescribed test was applied to data collected after 27 May 2006, with exactly the same reconstruction algorithms, energy calibration and quality cuts for event selection as in the exploratory scan. On 25 May 2007, 6 out of 8 events correlated, thus satisfying the prescription. In the independent data set collected up to 31 August 2007 there are 13 events with energy above $56 \mathrm{EeV}$, of which 8 have arrival directions closer than $3.1^{\circ}$ from the positions of AGN less than $75 \mathrm{Mpc}$ away, with 2.7 expected on average if the arrival directions were isotropic. The probability for this single configuration to happen by chance if the flux were isotropic (Eq. (2)) is $P=1.7 \times 10^{-3}$.

Following our search protocol and based on the independent data set alone, we reject the hypothesis of isotropy of the arrival directions of the highest-energy cosmic rays with at least $99 \%$ confidence level.

An alternative standard technique in sequential analysis could also have been used to monitor the evolution of the correlation signal: the sequential likelihood ratio test [30,31]. For the sequential test of AGN correlation, the likelihood ratio $R$ is given by the relative binomial probabilities of the isotropic (binomial parameter $p=0.21$ in our case) and anisotropic (binomial parameter $p_{1}>p$ ) cases. Since $p_{1}$ is not known, we integrate over $p<p_{1}<1$ to obtain the test ratio, $R$ as defined by Wald $[31,32]$

$R=\frac{\int_{p}^{1} p_{1}^{k}\left(1-p_{1}\right)^{N-k} \mathrm{~d} p_{1}}{p^{k}(1-p)^{N-k+1}}$.

The test rejects the isotropy hypothesis whenever $R \geqslant$ $(1-\beta) / \alpha$ (95 in our case) with the type I error $\alpha=1 \%$ and type II error $\beta=5 \%$ as previously specified.

This ratio test concluded at the rejection of the isotropy hypothesis when 7 out of the first 10 events of our independent data set correlated with AGN locations (see 


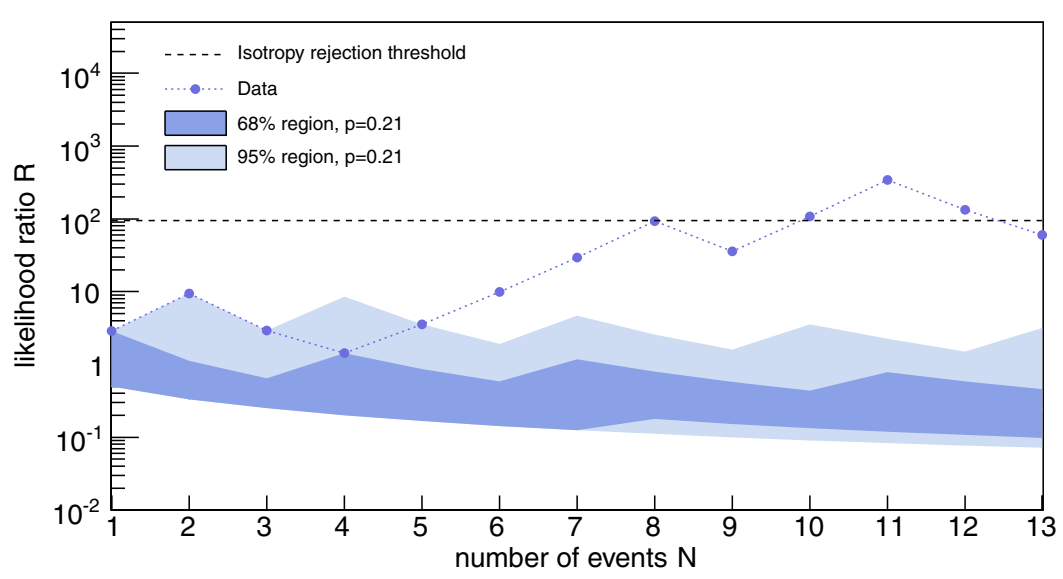

Fig. 1. Likelihood ratio $R$ as a function of the number of events observed in the prescribed test. The null hypothesis (isotropy) was rejected at the $99 \%$ likelihood threshold with 10 events. Shaded regions indicate expectations from isotropy at the $68 \%$ and $95 \%$ confidence limit.

Fig. 1, which also shows the subsequent evolution of the signal).

\section{The AGN correlation signal}

Having determined that an anisotropy exists according to an a priori search over an independent subset of the Auger data, we now consider results using the full data set (1 January 2004-31 August 2007) which allows us to obtain a more accurate measurement of the correlation signal. This data set, constructed using an updated version of our reconstruction algorithm (see Appendix A), contains 81 events with energy above $40 \mathrm{EeV}$ and zenith angle smaller than $60^{\circ}$, which satisfy the quality criteria given in Section 2.1 .

\subsection{Maximum correlation parameters}

Using the method described in Section 2.3 applied to the full data-set, we performed a scan within the range of parameters $1^{\circ} \leqslant \psi \leqslant 8^{\circ}, \quad 0 \leqslant z_{\max } \leqslant 0.024$ and $E_{\mathrm{th}} \geqslant 40 \mathrm{EeV}$. Catalogue-incompleteness prevents reliable exploration of higher redshifts. The scan in maximum angular distance is performed in steps of $0.1^{\circ}$, and the scan in maximum redshift $z_{\max }$ is done in steps of 0.001 .

The minimum probability for the hypothesis of isotropic arrival directions is found for the parameter set $z_{\max }=0.017$ $\left(D_{\max } \approx 71 \mathrm{Mpc}\right), \quad \psi=3.2^{\circ}$, and $E_{\mathrm{th}}=57 \mathrm{EeV}$. These results are statistically consistent with the results obtained from the earlier exploratory scan.

With these selected parameters, we find that 20 out of 27 cosmic ray events correlate with at least one of the 442 selected AGN (292 in the field of view of the Observatory), while only 5.6 are expected on average to do so if the flux were isotropic $(p=0.21)$. The respective cumulative binomial probability (Eq. (2)) of achieving this level of correlation from an isotropic distribution is $P_{\min }=4.6 \times 10^{-9}$. The chance probability that the observed correlation arose from an isotropic flux is much larger than $P_{\min }$, as already discussed in Section 2.3, because a scan was performed over a large parameter space to find the minimum of $P$.

To account for the effects of the scan we built simulated sets each with equal number of arrival directions ( 81 in our case) drawn from an isotropic flux in proportion to the relative exposure of the Observatory, and counted the fraction of simulated sets which had, anywhere in the parameter space and under the same scan, equal or smaller values of $P_{\min }$ than the minimum found in the data [18]. With this procedure, we obtained smaller or equal values of $P_{\min }$ in $10^{-5}$ of the simulated sets.

In Fig. 2 we present a sky map, in galactic coordinates, with circles of radius $3.2^{\circ}$ around each of the arrival directions of the 27 events with energy $E>57 \mathrm{EeV}$ detected by the Pierre Auger Observatory, along with asterisks at the positions of the $442 \mathrm{AGN}$ with redshift $z \leqslant 0.017$ in the $\mathrm{V}-\mathrm{C}$ catalogue. Each coloured band represents an equal integrated exposure which varies by about a factor of 3 between the lightest and darkest band. The number of AGN in each of those 6 bands is given in Table 2. The energies and arrival directions of the events are listed in Appendix A.

\subsection{Properties of the correlation signal}

In Fig. 3 we show one-dimensional plots of the probability $P$ as a function of each of the scan parameters with the other two held fixed at the values which lead to the absolute minimum probability.

We note that the energy threshold at which the correlation with nearby $\mathrm{AGN}$ is maximised, i.e. $E_{\mathrm{th}}=57 \mathrm{EeV}$, matches the energy range at which the flux measured by the Pierre Auger Observatory is $\sim 50 \%$ lower than would be expected from a power law extrapolation of the spectrum measured at lower energies [14]. This feature adds support to the interpretation that the correlation with relatively nearby sources is evidence for the GZK effect $[3,4]$, as will be discussed in Section 4.4 .

Relatively small values of $P$ occur for the energy threshold $E_{\mathrm{th}} \sim 60 \mathrm{EeV}$ for a range of maximum distances to 


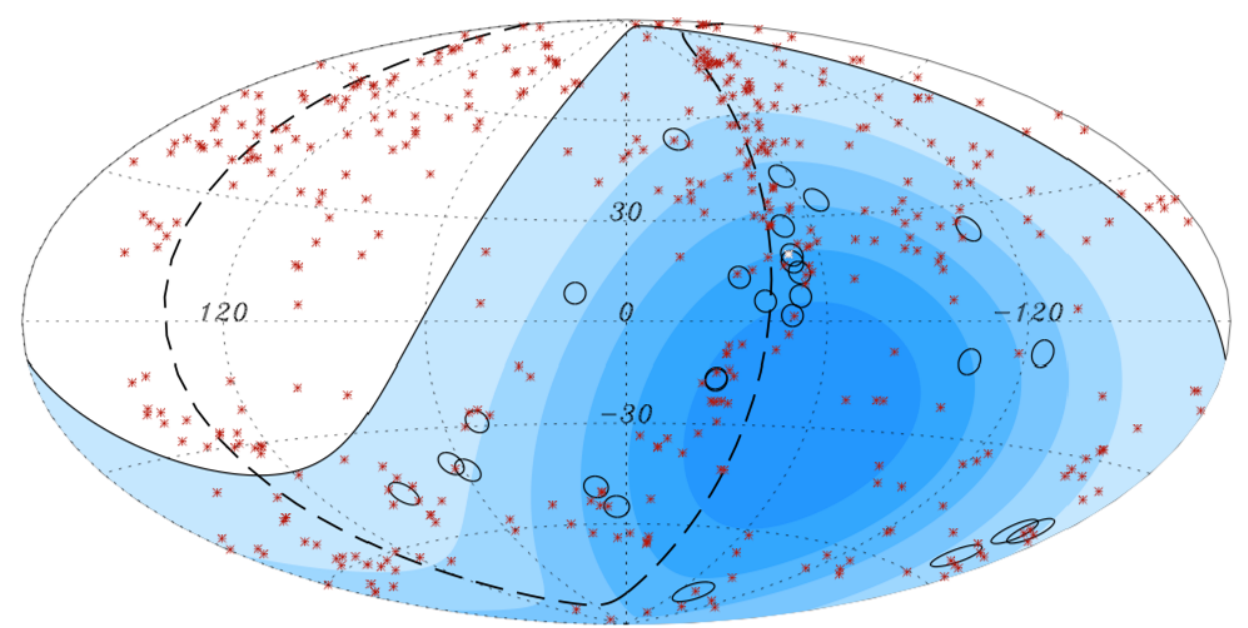

Fig. 2. Aitoff projection of the celestial sphere in galactic coordinates with circles of $3.2^{\circ}$ centred at the arrival directions of 27 cosmic rays detected by the Pierre Auger Observatory with reconstructed energies $E>57 \mathrm{EeV}$. The positions of the 442 AGN ( 292 within the field of view of the Observatory) with redshift $z \leqslant 0.017(D<71 \mathrm{Mpc})$ from the 12th edition of the catalogue of quasars and active nuclei [11] are indicated by asterisks. The solid line draws the border of the field of view for the southern site of the Observatory (with zenith angles smaller than $60^{\circ}$ ). The dashed line is, for reference, the super-galactic plane. Darker colour indicates larger relative exposure. Each coloured band has equal integrated exposure. Centaurus A, one of the closest AGN, is marked in white.

Table 2

Number of AGN with $z \leqslant 0.017$ in each of the exposure bands indicated in Fig. 2

\begin{tabular}{llll}
\hline Declination range & $\begin{array}{l}\text { Aperture } \\
\text { fraction }\end{array}$ & $\begin{array}{l}\text { Sky } \\
\text { fraction }\end{array}$ & $\begin{array}{l}\text { Number of } \\
\text { AGN }\end{array}$ \\
\hline$-90^{\circ}<\delta<-57.3^{\circ}$ & $1 / 6$ & 0.08 & 25 \\
$-57.3<\delta<-42.3^{\circ}$ & $1 / 6$ & 0.08 & 24 \\
$-42.3^{\circ}<\delta<-29.5^{\circ}$ & $1 / 6$ & 0.09 & 46 \\
$-29.5^{\circ}<\delta<-16.8^{\circ}$ & $1 / 6$ & 0.10 & 27 \\
$-16.8^{\circ}<\delta<-2.4^{\circ}$ & $1 / 6$ & 0.12 & 63 \\
$-2.4^{\circ}<\delta<24.8^{\circ}$ & $1 / 6$ & 0.23 & 107 \\
$24.8^{\circ}<\delta<90^{\circ}$ & 0 & 0.29 & 150 \\
\hline
\end{tabular}

Each of the top 6 bands represent $1 / 6$ of the total exposure, the corresponding fraction of the whole sky is also indicated. The last declination band represents the part of the sky outside the field of view of Auger for zenith angles $\theta<60^{\circ}$.

AGN between 50 and $100 \mathrm{Mpc}$ and for angular separations up to $6^{\circ}$. For instance, there is a local minimum with a value $P=8 \times 10^{-9}$ very close to that of the absolute minimum $\left(P_{\min }=4.6 \times 10^{-9}\right)$ for the set of parameters $\psi=4.8^{\circ}, z_{\max }=0.013\left(D_{\max }=55 \mathrm{Mpc}\right)$. With this set of parameters there are 22 events among the 27 with $E>57 \mathrm{EeV}$ that correlate with at least one of the 310 selected AGN, while only 7.4 were expected, on average, to do so by chance if the flux was isotropic $(p=0.28)$. With limited statistics, the parameters that minimise the probability $P$ should only be taken as indicative values of the relevant correlation scales.

AGN catalogues are likely to be incomplete near the galactic plane, where extinction from dust in the milky way reduces the sensitivity of observations. Moreover, cosmic rays that arrive close to the galactic plane are likely to have been deflected by the magnetic field in the disk more than those which arrive with higher galactic latitudes. These effects could have some impact upon the estimate of the strength and of the parameters that characterise
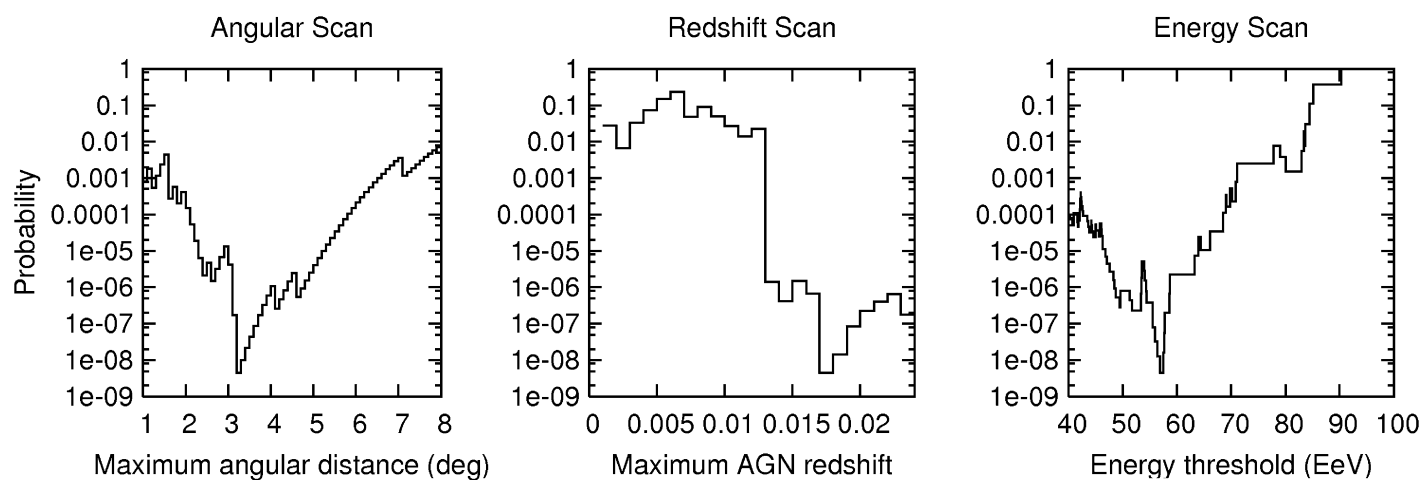

Fig. 3. Probability for the null hypothesis (isotropic distribution) vs. maximum angular distance $\psi$ (left), maximum AGN redshift $z_{\max }$ (centre), and threshold cosmic-ray energy $E_{\mathrm{th}}$ (right). In each case the other two parameters are held fixed at the values that lead to the absolute minimum probability $\left(\psi=3.2^{\circ}, z_{\max }=0.017, E_{\mathrm{th}}=57 \mathrm{EeV}\right)$. 


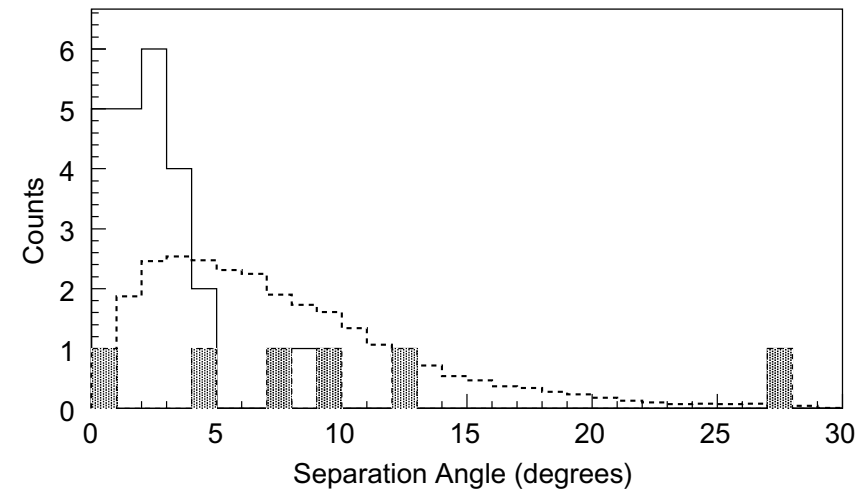

Fig. 4. Distribution of angular separations to the closest AGN within $71 \mathrm{Mpc}$. The 6 events with $|b| \leqslant 12^{\circ}$ have been shaded in grey. The average expectation for an isotropic flux is shown as the dashed line histogram.

the correlation. Catalogue incompleteness would weaken the measured strength of a true correlation.

In Fig. 4 we plot the distribution of angular separations between the arrival directions of the 27 highest-energy events and the position of the closest AGN with redshift $z \leqslant 0.017$. On this graph the 6 events with galactic latitudes $|b|<12^{\circ}$ have been shaded in grey. The two distributions are clearly distinct, a likely consequence of the incompleteness of the V-C catalogue at low galactic latitudes. The dashed line is, for comparison, the distribution expected, on average, from an isotropic flux modulated by the relative exposure of the Observatory.

We have performed a scan limited to events with galactic latitudes $|b|>12^{\circ}$. The minimum probability for the hypothesis of isotropic arrival directions occurs for the same parameters as without the cut in the galactic plane $\left(\psi=3.2^{\circ}, z_{\max }=0.017, E_{\mathrm{th}}=57 \mathrm{EeV}\right)$. The cut increases the strength of the correlation $\left(P_{\min }=1.7 \times 10^{-10}\right)$. Nineteen out of 21 arrival directions correlate with AGN positions while 5.0 are expected to do so by chance if the flux were isotropic. In other words, 5 of the 7 events which do not correlate with AGN positions arrive with galactic latitudes $|b|<12^{\circ}$.

A distribution of arrival directions of cosmic rays that shows evidence of anisotropy by correlation with a set of astrophysical objects is also expected to show evidence for anisotropy by auto-correlation. The degree of auto-correlation in the set of the 27 events with $E>57 \mathrm{EeV}$ is shown in Fig. 5, where we plotted the number of event pairs with angular separation smaller than a given value. Points represent the number of pairs in the data. Also shown are the mean number of pairs expected in simulated isotropic sets of 27 directions, distributed in proportion to the exposure of the Observatory. The error bars represent the dispersion of $90 \%$ of the simulations. Significant departures from isotropy are seen to occur at intermediate angular scales, between $9^{\circ}$ and $22^{\circ}$. This may be the consequence of a combination of clustering of events from individual sources in addition to effects of the non-uniform distribution of the sources themselves [26].

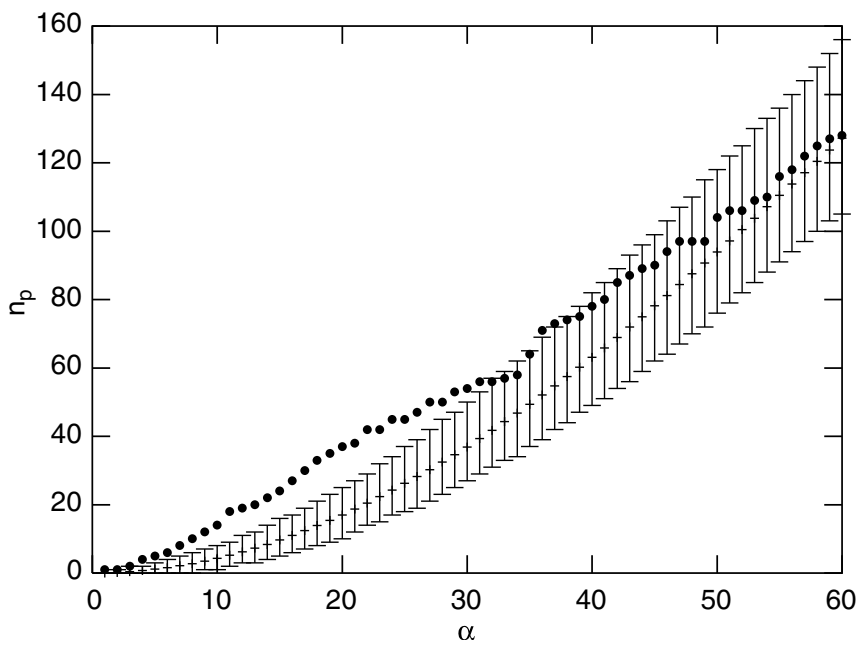

Fig. 5. Number of pairs as a function of maximum separation angle $\alpha$ for the 27 events with $E>57 \mathrm{EeV}$ (points) and average expectation for an isotropic flux. The error bars on the isotropic expectations represent the $90 \%$ confidence limit dispersion.

To compare the auto-correlation function of the data to that expected from the AGN distribution in the V-C catalogue we must restrict ourselves to the regions where the catalogue is reasonably complete, e.g. outside of the galactic plane. In Fig. 6 we plot the number of pairs in the data as a function of the separation angle restricted to the 21 events with $E>57 \mathrm{EeV}$ and galactic latitudes $|b|>12^{\circ}$. Also shown is the average distribution expected in sets of 21 directions chosen at random (in proportion to the relative exposure of the Observatory) from the positions of AGN in the V-C catalogue with redshift $z \leqslant 0.017$ and $|b|>12^{\circ}$. The error bars in the plots indicate the results in $90 \%$ of the simulated sets. The distribution of pairs in the data are in all cases within those results.

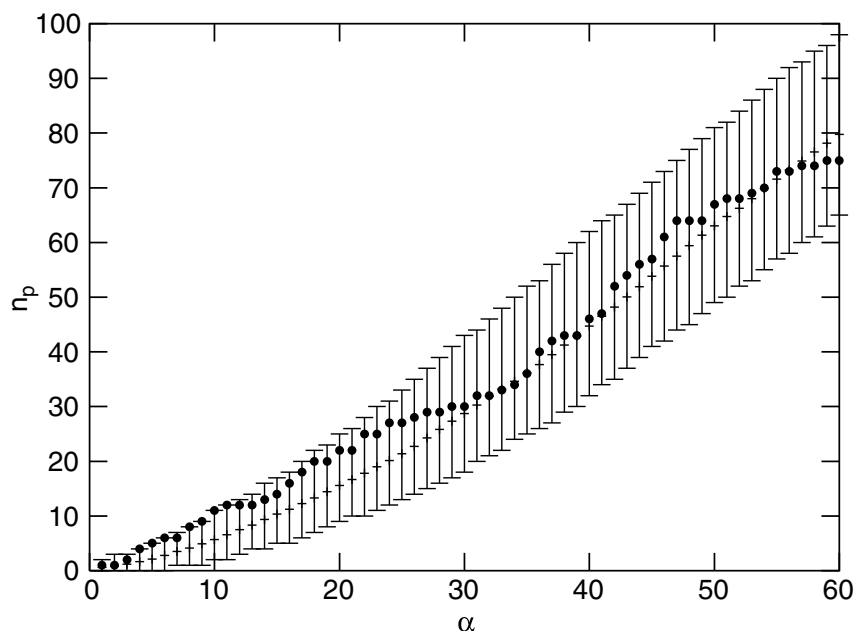

Fig. 6. Number of pairs as a function of maximum separation angle $\alpha$ for the subset of 21 events with $E>57 \mathrm{EeV}$ and $|b|>12^{\circ}$ (points) and average expectation for AGN in the V-C catalogue with $z \leqslant 0.017$ and the same cut in galactic latitude. The error bars on the AGN expectations represent the $90 \%$ confidence limit dispersion. 
Anisotropy in the distribution of arrival directions can also be characterised by the multipole moments of an expansion in spherical harmonics $Y_{\ell, m}$. With partial coverage of the sky the application of this method is not straightforward [33]. A method has been developed [34] to cope with partial sky coverage that allows a bound to be placed, with a given confidence level, to the minimum multipole order necessary to account for the observed distribution. The application of this method to the arrival directions of the events with energies above $50 \mathrm{EeV}$ in our data set shows that a multipole order $\ell \geqslant 5$ is necessary to describe their distribution with $99 \%$ confidence level. The arrival directions of events with energy between 40 and $50 \mathrm{EeV}$ do not show evidence of anisotropy with this method. This result is consistent with the sharp decrease of the correlation with AGN positions at lower energies.

\section{Discussion}

In the previous sections we have demonstrated the anisotropy of the highest-energy cosmic rays and have derived a set of parameters that maximises the correlation with the AGN sample from the 12th edition of the V-C catalogue. In this section, based on those parameters and the data set that maximises the correlation, we discuss some possible implications of the observed signal.

\subsection{Lower limit on the number of sources}

If ultra high energy cosmic rays come from a large number of dim sources, the number of pairs of events (doublets) coming from one source is expected to be much smaller than the number of singlets. On the other hand, if they come from a small number of bright sources, the ratio of doublets to singlets is expected to be larger. It is then possible to put a lower limit on the number of sources based on the ratio of doublets to singlets. The minimum number of sources, $S$, results for the case in which all the sources have the same apparent luminosity [35]. If sources are steady, cosmic rays accelerated by one source at different times are statistically independent and the detection can be considered as a Poisson process. Then the probability that one source accelerates $n$ particles is given by $P(n)=v^{n} \mathrm{e}^{-v} / n$ !, with $v$ the mean number of events expected from one source. The mean number of expected singlets from $S$ sources is $n_{1}=$ $S \times P(1)=S v \mathrm{e}^{-v}$ and that of doublets is $n_{2}=S \times$ $P(2)=S v^{2} \mathrm{e}^{-v} / 2$. It is thus possible to estimate the number of sources $S$ as

$S \sim \frac{n_{1}^{2}}{2 n_{2}} \mathrm{e}^{\left(2 n_{2}\right) / n_{1}}$

Within the 27 highest-energy events there are 6 pairs with separation smaller than the correlation angular scale of $6^{\circ}$, while 1.6 are expected by chance in an isotropic flux. Taking $n_{2}=6-1.6=4.4$ and $n_{1}=27-2 n_{2}=18.2$, we obtain a lower limit for the number of sources $S \geqslant 61$.
Note that this is a bound for mean expectations, but could have large fluctuations with the present small statistics. Also, it was derived under the unrealistic assumption of equal flux on Earth for all sources. Assuming instead equal intrinsic luminosity in cosmic rays the mean number of sources becomes larger by a factor of order $n_{1} / n_{2}$ [35]. The lower bound could also increase if the sources had significant clustering of their own on the same angular scale as the clustering of events. In either case, this lower limit does not contradict the hypothesis that nearby AGN are the sources.

\subsection{Signal dependence on energy}

We have studied the dependence on energy of the correlation of our data set with the AGN from the V-C catalogue. In an approach similar to the one developed in [36] we constructed a smoothed density map from the V$\mathrm{C}$ catalogue and used it to compute the log-likelihood of any event sample. We then compared the result for the data to that for simulated samples of the same size, either drawn from an isotropic distribution of arrival directions or from the smoothed density map itself, in both cases modulated by the relative acceptance of the Observatory.

From the density map of the V-C catalogue, smoothed on a given angular scale and limited to within $100 \mathrm{Mpc}$, it is possible to calculate the average log-likelihood of an event sample

$\mathrm{LL}=\frac{1}{N} \sum_{k=1}^{N} \log \left(\rho_{k}\right)$

where the sum runs over the $N$ events of the sample under consideration and $\rho_{k}$ is the map density in the direction of the event $k$.

In Fig. 7 we present the values of LL (dots) from our data in $10 \mathrm{EeV}$ energy intervals. We used events with galactic

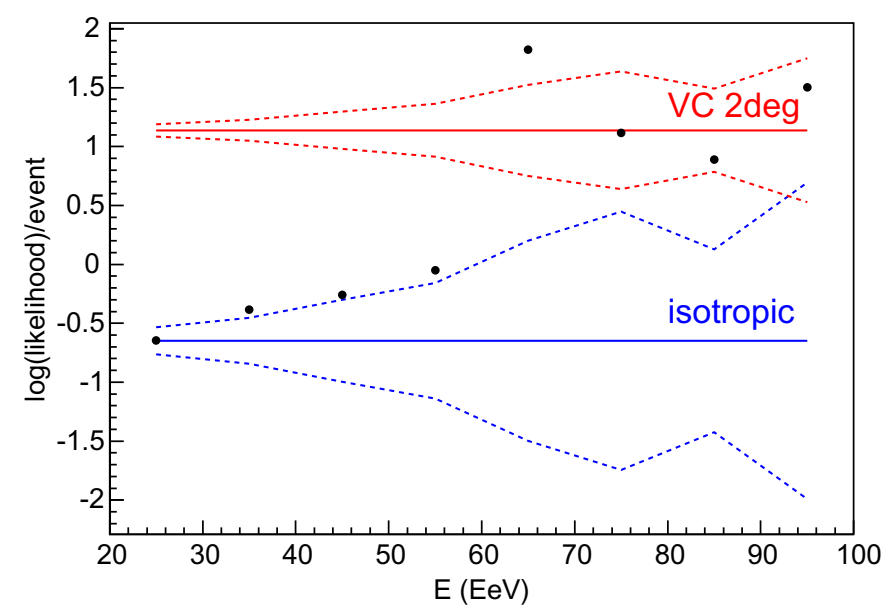

Fig. 7. Comparison of the average log-likelihood per event in different energy intervals (calculated using the smoothed distribution of AGN) between real data and simulated samples (bottom lines isotropic distribution, top lines AGN distribution from V-C catalog). 
latitudes $|b|>12^{\circ}$, and compared them with the average expected from samples of similar size and generated either according to an isotropic distribution (bottom (blue) line) or to the distribution of AGN within $100 \mathrm{Mpc}$ smoothed with Gaussian windows of $2^{\circ}$ (top (red) line). The dashed lines represent the $1 \sigma$ interval around the mean value, based on the statistics of the real data. The data are compatible with a distribution that follows that of AGN at high energies with an abrupt transition towards an isotropic distribution below $60 \mathrm{EeV}$.

\subsection{Source identification}

The high degree of correlation that we observe can certainly serve as a strong indication that AGN may well be the sources of ultra-high energy cosmic rays. However, this result is not yet a proof.

In particular, we know that the distribution of matter (visible and dark) in our local Universe (within $100 \mathrm{Mpc}$ ) is strongly non-uniform, and that AGN are correlated with this non-uniformity. We are therefore motivated to see if our data provide sufficient information to determine if the correlation signal is unambiguously associated with AGN or if they are simply acting as tracers of some other set of sources with a similar distribution.

In a specific study, we have compared the arrival directions of our highest-energy events with the distribution expected from different source models using a likelihood test. The test compared isotropic distributions, distributions of galaxies from the IRAS PSCz [37] catalogue and distributions of $\mathrm{AGN}$ from the $\mathrm{V}-\mathrm{C}$ catalogue, at different angular scales and using different horizon depths (see 4.4). In addition to confirming that our data are incompatible with an isotropic distribution these studies showed that they are best represented by a small angular smoothing within a relatively nearby horizon $(100 \mathrm{Mpc})$ of the AGN from the V-C catalogue [36].

The local spatial distribution of AGN is correlated with the distribution of other astronomical objects that are potential sources of cosmic rays, such as rich clusters of galaxies and star-burst galaxies (which could host a large fraction of $\gamma$-ray bursts). We have tested, with the same scan method that we applied to the V-C catalogue, whether there is a significant correlation with the Abell clusters of galaxies (an optical survey) [38] and with the clusters in the X-ray surveys REFLEX [39] and NORAS [40]. We also tested for correlation against a catalogue of star-burst galaxies [41]. We did not find significant correlation in these searches with our present data set.

It is plausible that only a subclass of AGN in the V-C catalogue are the sources of the highest-energy cosmic rays. With our present relatively small data set it is difficult to pinpoint distinctive properties of the AGN that are close to their arrival directions, or to draw firm conclusions about patterns in their redshift distribution. It is worth noting, as is clearly visible in Fig. 2, the striking alignment of several events close to the super-galactic plane. Two of the events have arrival directions less than $3^{\circ}$ away from Centaurus A, one of the closest AGN.

\subsection{The GZK horizon}

The correlation observed is consistent with the hypothesis that the highest-energy cosmic rays that arrive on earth are predominantly produced in relatively nearby AGN, within the distance over which the GZK effect $[3,4,42]$ does not significantly attenuate their flux.

The "GZK horizon" may be defined as the distance from the Earth which contains the sources that produce $90 \%$ of the protons that arrive with energies above a given threshold. Under the idealisation of uniformly distributed sources of equal intrinsic cosmic ray luminosity and a conventional spectral index, the horizon computed in the continuous energy loss approximation is about $90 \mathrm{Mpc}$ for protons that arrive with energies above $80 \mathrm{EeV}$ and about $200 \mathrm{Mpc}$ for energies above $60 \mathrm{EeV}$ [43]. Deviations of the horizon scale from the estimates above are expected, in particular due to local departures of the sources from uniformity in spatial distribution, intrinsic luminosity, and spectral features.

The largest departure from isotropic expectations (minimum value of the probability $P$ ) in the complete data set was found to be due to correlation with AGN at a distance smaller than $71 \mathrm{Mpc}$ and for cosmic rays with energies above $57 \mathrm{EeV}$. However, relatively small values of $P$ occur for this energy threshold for a range of maximum distances to AGN between 50 and $100 \mathrm{Mpc}$.

If these numbers were to be taken at face value, an upward shift in the energy calibration of $\sim 30 \%$, as suggested in some simulations of the reconstruction of the shower energies [44], would lead to a better agreement between the maximum AGN distance $D_{\max }$ that minimises the probability $P$ and the theoretical expectations based on the idealised GZK attenuation. However, while we expect $D_{\max }$ to be comparable to the GZK horizon scale, the relation is not a simple one. An accidental correlation with foreground AGN different from the actual source may induce some bias in the value of $D_{\max }$ toward smaller maximum source distances. The $P$ minimisation method has non-uniform sensitivity over the range of parameters explored. Incompleteness of the $\mathrm{V}-\mathrm{C}$ catalogue prevents a reliable exploration beyond approximately $100 \mathrm{Mpc}$. Furthermore, as mentioned above, a realistic estimate of the horizon scale depends on several unknown features. A large local over-density of sources would reduce its value. The distribution of intensity and spectral features of the dominant sources also has an effect on the horizon scale.

Regarding the possibility that the cosmic rays injected at the sources are heavy nuclei, attenuated mainly by photodisintegration processes, one may note that nuclei of the iron group have horizons only slightly smaller than the proton horizons, but intermediate mass nuclei $(A \simeq$ 20-40) have significantly smaller horizons (e.g. the horizon for a threshold energy of $60 \mathrm{EeV}$ is about $60 \mathrm{Mpc}$ for ${ }^{28} \mathrm{Si}$ nuclei [43]). The smaller horizon for decreasing nuclear 

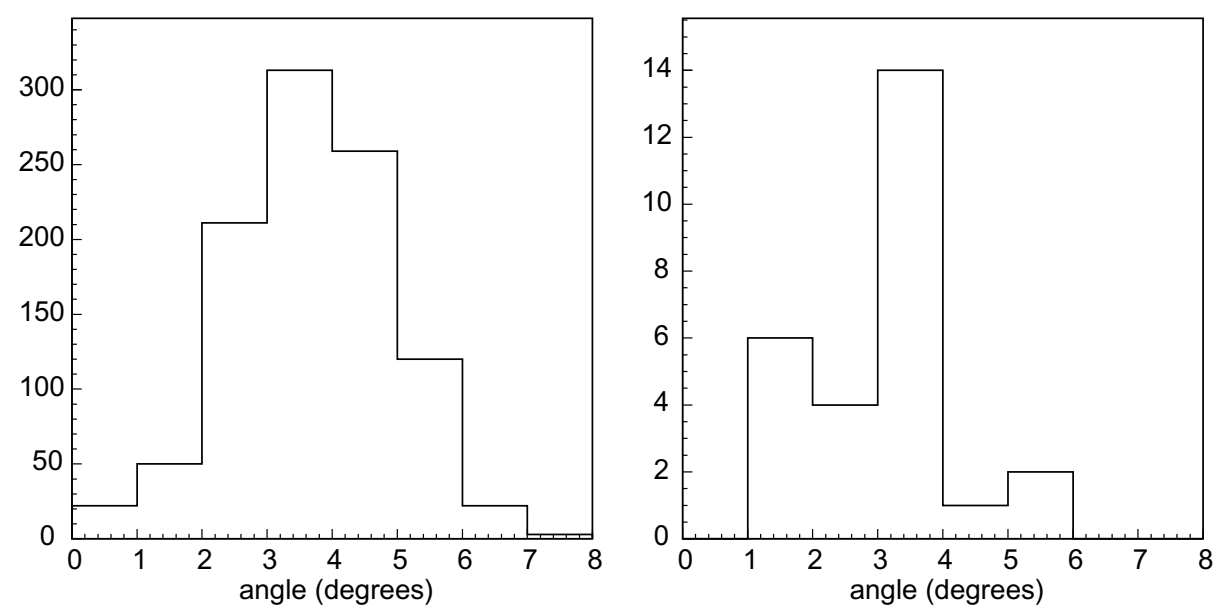

Fig. 8. Distribution of the deflections for protons in the BSS-S model of the galactic magnetic field. Left panel: 1000 directions drawn from an isotropic flux in proportion to the exposure of the Observatory, for $E=60 \mathrm{EeV}$. Right panel: deflections of the 27 arrival directions of the observed events with $E>57 \mathrm{EeV}$.

mass is due to the corresponding decrease in the threshold required to excite the giant-dipole resonance for photodisintegration.

\subsection{Effect of the magnetic fields}

A cosmic ray with charge $Z e$ that travels a distance $D$ in a regular magnetic field $\mathbf{B}$ is deflected by an angle $\delta$ given by

$\delta \simeq 2.7^{\circ} \frac{60 \mathrm{EeV}}{E / Z}\left|\int_{0}^{D}\left(\frac{\mathrm{d} \mathbf{x}}{\mathrm{kpc}} \times \frac{\mathbf{B}}{3 \mu \mathrm{G}}\right)\right|$

If the regular galactic magnetic field has a strength of a few $\mu \mathrm{G}$ with a coherence scale of order $\sim 1 \mathrm{kpc}$, as in some models [45], the deflection is expected to be a few degrees for protons with $E>60 \mathrm{EeV}$. In such models, the angular scale of the correlation we observed is consistent with the size of the deflections expected to be imprinted upon protons by the galactic magnetic field.

The precise amount of the deflection is very dependent on each specific arrival direction. We have evaluated numerically the deflections imparted in a conventional regular galactic magnetic field model [45] for sets of arrival directions uniformly distributed according to the Pierre Auger Observatory relative exposure. Anti-particles were backtracked in the galactic regular magnetic field to a distance of $20 \mathrm{kpc}$ away from the Galactic Centre (where the field strength is already very small). At this point the angle between the initial (as measured on the earth) and final velocity vectors was calculated. The result is shown in the left panel of Fig. 8 in the special case of the BSS-S model ${ }^{2}$ (without $B_{z}$ component) for $E=60 \mathrm{EeV}$ protons. The deflections scale approximately as $Z \times(60 \mathrm{EeV} / E)$ for

\footnotetext{
${ }^{2}$ In fact, we have smoothed the original BSS-S model of [45] as described in [46] in order to avoid the discontinuities present in the original model.
}

other energies and electric charges (the scaling is rigorous only for very small deflections and a uniform field). In the right panel of the figure we show the distribution of deflections for protons in the case of the 27 arrival directions of the events with $E>57 \mathrm{EeV}$, as computed for each using its reconstructed energy.

Models of the regular component of the galactic magnetic field [47] outline its basic features, but cannot be expected to provide a complete picture nor a realistic value for every direction. It is, for example, possible to do the exercise of "correcting" the observed arrival directions to undo the deviation imparted by the galactic magnetic field, but current models are not expected to be accurate enough to allow us to draw reliable conclusions from such analyses. Nonetheless, the results shown in Fig. 8 provide a reasonable estimate of the typical deflections to be expected. They are consistent with the angular scale of the observed correlation with AGN. Therefore, if the BSS-S model is a fair representation of the general features of the regular galactic magnetic field, then the correlation observed in the data would be unlikely if the primary composition of the cosmic rays reaching us were much heavier than protons. Note that this does not preclude the possibility that the source emits heavy nuclei, which could disintegrate along their journey, so that the lighter fragments are those deflected by the galactic magnetic field.

It will be possible in the future to compare various models for the galactic magnetic field with the pattern of orientation and size of the deviation between the observed arrival directions of the events and potential AGN sources. However, our present data set is not large enough to perform such an analysis reliably.

The angular scale of the observed correlation also implies that intergalactic magnetic fields along the line of sight to the sources do not in general deviate cosmic ray trajectories by much more than a few degrees. The rootmean-square deflection imprinted upon the trajectories of 
cosmic rays with charge $Z e$ as they travel a distance $D$ in a turbulent magnetic field with coherence length $L_{\mathrm{c}}$ is

$\delta_{\mathrm{rms}} \approx 4^{\circ} \frac{60 \mathrm{EeV}}{E / Z} \frac{B_{\mathrm{rms}}}{10^{-9} \mathrm{G}} \sqrt{\frac{D}{100 \mathrm{Mpc}}} \sqrt{\frac{L_{\mathrm{c}}}{1 \mathrm{Mpc}}}$

There is no measurement of the intergalactic magnetic fields except at the centres of rich galaxy clusters. Numerical simulations of those fields give a wide range of possible deflections from negligible [48] to very large [49]. The correlation observed can be used to constrain models of turbulent intergalactic magnetic fields, which must be such that in most directions $B_{\mathrm{rms}} \sqrt{L_{\mathrm{c}}} \leqslant 10^{-9} \mathrm{G} \sqrt{\mathrm{Mpc}}$ within our "GZK horizon".

Finally, there are possible biases in the determination of the relevant angular scale of the deflection. The active galaxy closest to the arrival direction of a cosmic ray is not necessarily the source responsible for it. This could lead to an underestimate of the deflection involved. In principle it could also happen, due to catalogue incompleteness or because the source is something else, that an AGN found to correlate with a particular event is further away than the actual source, thus overestimating the deflection angle.

\subsection{The acceleration sites}

Acceleration sites in the active galaxies that correlate with events above $57 \mathrm{EeV}$ are promising candidate sources of high energy cosmic rays, but other possible sites cannot be ruled out with the present limited statistics. (For a recent summary of proposed acceleration sites, see e.g. [50].) The observed correlation shows that ultra-high energy cosmic ray sources are extra-galactic with an angular distribution similar to that of AGN within $\sim 71 \mathrm{Mpc}$ and that the primaries are most likely protons that suffer losses due to interactions with the cosmic background radiation. These results rule out models for the origin of cosmic rays that place observed sources predominantly in our Galaxy, such as galactic compact objects (young neutron stars [51], pulsars [52], and black holes), and $\gamma$-ray bursts [53]. Models where sources are located in the galactic halo are also ruled out, such as the decay of super-heavy dark matter particles [54-56], which are already highly constrained by the Auger limit on the fraction of photon primaries at high energies $[57,58]$. Top-down models based on topological defects [59-61] need to have a spatial distribution consistent with the local matter distribution to avoid being excluded as significant sources of cosmic rays. Such models are also constrained by the photon and neutrino limits [58].

The large-scale structure distribution of matter, which is traced by normal galaxies, has a similar spatial distribution to the local AGN. Therefore, acceleration sites in galaxies with inactive nuclei cannot be excluded at the present, including those based on extra-galactic compact objects [62], quasar remnants [63], galactic winds in star-bursts galaxies [64], and $\gamma$-ray bursts $[65,66]$. In contrast, acceleration models in massive clusters of galaxies, such as cluster accretion shocks [67,68], are challenged by the observed correlation. Massive clusters are rare within $100 \mathrm{Mpc}$ when compared to the number of observed events and there is a paucity of events from the direction of Virgo, the nearest sizeable cluster of galaxies.

AGN have long been suggested as likely accelerators of cosmic rays $[8,9,69]$. The case for active galaxies as likely sources is based on the power available from the central black hole. AGN are powered by the accretion of matter onto a super-massive black hole (with masses in the range $10^{6}-10^{8} \mathrm{M}_{\odot}$ ) at the centre of the galaxy. A number of mechanisms have been proposed that utilise different regions and properties of this system to accelerate cosmic rays to ultra-high energies. Acceleration based on the central regions face the challenge of energy losses in the radiation field that surrounds the central black hole and accretion disk. Alternative acceleration sites include jets $[70,71]$ and radio lobes [72] that are associated with the most luminous AGN.

AGN with prominent radio lobes are rare and do not follow the observed spatial distribution of the observed correlated AGN. The one exception is Cen A, at only 3.4 Mpc [73], which has been proposed as a site for cosmic ray acceleration [74]. It displays jets, radio lobes which extend over a scale of about $10^{\circ}$ along the super-galactic plane, and a variable compact radio nucleus. Two events correlate with the nucleus position while several lie in the vicinity of the radio lobe extension along the super-galactic plane (see Fig. 2). The most prominent radio galaxy in our GZK neighbourhood is M87, which does not correlate with any observed event above $57 \mathrm{EeV}$ thus far but the coverage of the southern Auger Observatory is almost a factor of 3 lower in this direction of the sky than in the direction of Cen A. Of the remaining 18 correlating events, 15 have Seyfert galaxies as the closest AGN in angular separation.

A significant increase in ultra-high energy cosmic ray statistics combined with searches for counterparts in a multi-wavelength and multi-messenger campaign should improve our ability to distinguish if AGN are the sources of cosmic rays or tracers of the sources. If future data select AGN as hosts of cosmic ray accelerators, the type of AGN selected, together with spectral and composition information, should help distinguish between proposed AGN acceleration mechanisms.

\section{Conclusions}

Anisotropy has been established with more than 99\% confidence level in the arrival directions of events with energy above $\sim 60 \mathrm{EeV}$ detected by the Pierre Auger Observatory. These events correlate over angular scales of less than $6^{\circ}$ with the directions towards nearby $(D<100 \mathrm{Mpc})$ AGN.

The observed correlation demonstrates the extra-galactic origin of the highest-energy cosmic rays. It is consistent with the hypothesis that cosmic rays with energies above $\sim 60 \mathrm{EeV}$ are predominantly protons that come from 
AGN within our "GZK horizon". This provides evidence that the observed steepening of the cosmic ray spectrum at the highest-energies is due to the "GZK effect", and not to acceleration limits at the sources.

It is possible that the sources are other than AGN, as long as their local distribution is sufficiently correlated with them. Unequivocal identification of the sources requires a larger data set, such as the Pierre Auger Observatory will gather in a few years. In particular, one could use the fact that angular departures of the events from an individual source due to magnetic deflections should decrease in inverse proportion to the energy of the cosmic ray. The observation of such angle/energy correlation in clusters of events could be exploited to locate the source position unambiguously with high accuracy. This could also provide at the same time valuable and unique information about the magnetic fields along the line of sight.

We have shown that astronomy of charged particles is indeed feasible at the highest-energies and that in the next few years we can hope for unambiguous identification of sources of cosmic rays.

\section{Acknowledgement}

The successful installation and commissioning of the Pierre Auger Observatory would not have been possible without the strong commitment and effort from the technical and administrative staff in Malargüe.

We are very grateful to the following agencies and organizations for financial support: Comisión Nacional de Energía Atómica, Fundación Antorchas, Gobierno De La Provincia de Mendoza, Municipalidad de Malargüe, NDM Holdings and Valle Las Leñas, in gratitude for their continuing cooperation over land access, Argentina; the Australian Research Council; Conselho Nacional de Desenvolvimento Científico e Tecnológico (CNPq), Financiadora de Estudos e Projetos (FINEP), Fundação de Amparo à Pesquisa do Estado de Rio de Janeiro (FAPERJ), Fundação de Amparo à Pesquisa do Estado de São Paulo (FAPESP), Ministério de Ciência e Tecnologia (MCT), Brazil; Ministry of Education, Youth and Sports of the Czech Republic; Centre de Calcul IN2P3/CNRS, Centre National de la Recherche Scientifique (CNRS), Conseil Régional Ile-de-France, Département Physique Nucléaire et Corpusculaire (PNC-IN2P3/CNRS), Département Sciences de l'Univers (SDU-INSU/CNRS), France; Bundesministerium für Bildung und Forschung (BMBF), Deutsche Forschungsgemeinschaft (DFG), Finanzministerium Baden-Württemberg, Helmholtz-Gemeinschaft Deutscher Forschungszentren (HGF), Ministerium für Wissenschaft und Forschung, Nordrhein-Westfalen, Ministerium für Wissenschaft, Forschung und Kunst, BadenWürttemberg, Germany; Istituto Nazionale di Fisica Nucleare (INFN), Ministero dell'Istruzione, dell'Università e della Ricerca (MIUR), Italy; Consejo Nacional de Ciencia y Tecnología (CONACYT), Mexico; Ministerie van Onderwijs, Cultuur en Wetenschap, Nederlandse
Organisatie voor Wetenschappelijk Onderzoek (NWO), Stichting voor Fundamenteel Onderzoek der Materie (FOM), Netherlands; Ministry of Science and Higher Education, Grant Nos. 1 P03 D 014 30, N202 090 31/0623, and PAP/218/2006, Poland; Fundação para a Ciência e a Tecnologia, Portugal; Ministry for Higher Education, Science, and Technology, Slovenian Research Agency, Slovenia; Comunidad de Madrid, Consejería de Educación de la Comunidad de Castilla La Mancha, FEDER funds, Ministerio de Educación y Ciencia, Xunta de Galicia, Spain; Science and Technology Facilities Council, United Kingdom; Department of Energy, Contract No. DE-AC02-07CH 11359, National Science Foundation, Grant No. 0450696, The Grainger Foundation USA; ALFA-EC/ HELEN, European Union 6th Framework Program, Grant No. MEIF-CT-2005-025057, and UNESCO.

\section{Appendix A. Event list}

Here we list the 27 events recorded from 1 January 2004 until 31 August 2007 with energy in excess of $57 \mathrm{EeV}$. We have indicated the date of observation (year and Julian day), the zenith angle, the shower size at $1000 \mathrm{~m}$ from the core $S(1000)$, the energy in $\mathrm{EeV}$, the equatorial coordinates (RA, Dec) and the galactic coordinates (longitude, latitude). Events that correlate within $3.2^{\circ}$ of AGN with redshift $z \leqslant 0.017$ are marked with a star. The dashed horizontal line indicates the beginning of the prescribed test of Section 2.

The quoted energy is derived from a calibration procedure where the shower size is compared to the energy measured by the FD. This energy calibration, based on the sample of hybrid events analysed at the time of the prescription, was used for the whole data set for consistency. The smaller uncertainty on the energy calibration curve expected from the increased statistics of hybrid events, as well as improvements in the systematic uncertainty of the FD energy scale, may lead to revised energies in future publications. Thus, we also include the shower size $S(1000)$ at $1000 \mathrm{~m}$ from the reconstructed core, as it is the shower parameter that is directly measured from the individual SD signals in the event. This parameter is almost independent of the shower lateral distribution function used in the reconstruction procedure (within 10\%). The uncertainty in $S$ resulting from the adjustment of the shower size, the conversion to a reference angle, the fluctuation from shower-to-shower and the calibration curve amounts to about $18 \%$. The absolute energy scale is given by the fluorescence measurements and has a systematic uncertainty of $22 \%$ [15].

Note that the energies and arrival directions given in this list correspond to the analysis of the full data set which used a slightly different reconstruction package than the one used for the original scan and the prescribed test of Section 2. In particular improvements made in the SD tank calibration have very slightly modified the energy and arrival directions. 


\begin{tabular}{|c|c|c|c|c|c|c|c|c|}
\hline Year & Julian day & $\theta$ & $S(1000)$ & $E(\mathrm{EeV})$ & RA & Dec & Longitude & Latitude \\
\hline 2004 & 125 & 47.7 & 252 & 70 & $267.1^{\circ}$ & $-11.4^{\circ}$ & $15.4^{\circ}$ & $8.4^{\circ}$ \\
\hline 2004 & 142 & 59.2 & 212 & 84 & $199.7^{\circ}$ & $-34.9^{\circ}$ & $-50.8^{\circ}$ & $27.6^{\circ *}$ \\
\hline 2004 & 282 & 26.5 & 328 & 66 & $208.0^{\circ}$ & $-60.3^{\circ}$ & $-49.6^{\circ}$ & $1.7^{\circ *}$ \\
\hline 2004 & 339 & 44.7 & 316 & 83 & $268.5^{\circ}$ & $-61.0^{\circ}$ & $-27.7^{\circ}$ & $-17.0^{\circ *}$ \\
\hline 2004 & 343 & 23.4 & 323 & 63 & $224.5^{\circ}$ & $-44.2^{\circ}$ & $-34.4^{\circ}$ & $13.0^{\circ *}$ \\
\hline 2005 & 54 & 35.0 & 373 & 84 & $17.4^{\circ}$ & $-37.9^{\circ}$ & $-75.6^{\circ}$ & $-78.6^{\circ *}$ \\
\hline 2005 & 63 & 54.5 & 214 & 71 & $331.2^{\circ}$ & $-1.2^{\circ}$ & $58.8^{\circ}$ & $-42.4^{\circ *}$ \\
\hline 2005 & 81 & 17.2 & 308 & 58 & $199.1^{\circ}$ & $-48.6^{\circ}$ & $-52.8^{\circ}$ & $14.1^{\circ *}$ \\
\hline 2005 & 295 & 15.4 & 311 & 57 & $332.9^{\circ}$ & $-38.2^{\circ}$ & $4.2^{\circ}$ & $-54.9^{\circ *}$ \\
\hline 2005 & 306 & 40.1 & 248 & 59 & $315.3^{\circ}$ & $-0.3^{\circ}$ & $48.8^{\circ}$ & $-28.7^{\circ *}$ \\
\hline 2005 & 306 & 14.2 & 445 & 84 & $114.6^{\circ}$ & $-43.1^{\circ}$ & $-103.7^{\circ}$ & $-10.3^{\circ}$ \\
\hline 2006 & 35 & 30.8 & 398 & 85 & $53.6^{\circ}$ & $-7.8^{\circ}$ & $-165.9^{\circ}$ & $-46.9^{\circ *}$ \\
\hline 2006 & 55 & 37.9 & 255 & 59 & $267.7^{\circ}$ & $-60.7^{\circ}$ & $-27.6^{\circ}$ & $-16.5^{\circ *}$ \\
\hline 2006 & 81 & 34.0 & 357 & 79 & $201.1^{\circ}$ & $-55.3^{\circ}$ & $-52.3^{\circ}$ & $7.3^{\circ}$ \\
\hline 2006 & 185 & 59.1 & 211 & 3 & $350.0^{\circ}$ & $9.6^{\circ}$ & $\begin{array}{r}88.8^{\circ}\end{array}$ & $-47.1^{\circ *}$ \\
\hline 2006 & 296 & 54.0 & 208 & 69 & $52.8^{\circ}$ & $-4.5^{\circ}$ & $-170.6^{\circ}$ & $-45.7^{\circ *}$ \\
\hline 2006 & 299 & 26.0 & 344 & 69 & $200.9^{\circ}$ & $-45.3^{\circ}$ & $-51.2^{\circ}$ & $17.2^{\circ *}$ \\
\hline 2007 & 13 & 14.3 & 762 & 148 & $192.7^{\circ}$ & $-21.0^{\circ}$ & $-57.2^{\circ}$ & $41.8^{\circ}$ \\
\hline 2007 & 51 & 39.2 & 247 & 58 & $331.7^{\circ}$ & $2.9^{\circ}$ & $63.5^{\circ}$ & $-40.2^{\circ *}$ \\
\hline 2007 & 69 & 30.4 & 332 & 70 & $200.2^{\circ}$ & $-43.4^{\circ}$ & $-51.4^{\circ}$ & $19.2^{\circ * *}$ \\
\hline 2007 & 84 & 17.3 & 340 & 64 & $143.2^{\circ}$ & $-18.3^{\circ}$ & $-109.4^{\circ}$ & $23.8^{\circ *}$ \\
\hline 2007 & 145 & 23.9 & 392 & 78 & $47.7^{\circ}$ & $-12.8^{\circ}$ & $-163.8^{\circ}$ & $-54.4^{\circ *}$ \\
\hline 2007 & 186 & 44.8 & 248 & 64 & $219.3^{\circ}$ & $-53.8^{\circ}$ & $-41.7^{\circ}$ & $5.9^{\circ}$ \\
\hline 2007 & 193 & 18.0 & 469 & 90 & $325.5^{\circ}$ & $-33.5^{\circ}$ & $12.1^{\circ}$ & $-49.0^{\circ *}$ \\
\hline 2007 & 221 & 35.3 & 318 & 71 & $212.7^{\circ}$ & $-3.3^{\circ}$ & $-21.8^{\circ}$ & $54.1^{\circ *}$ \\
\hline 2007 & 234 & 33.2 & 365 & 80 & $185.4^{\circ}$ & $-27.9^{\circ}$ & $-65.1^{\circ}$ & $34.5^{\circ}$ \\
\hline 2007 & 235 & 42.6 & 276 & 69 & $105.9^{\circ}$ & $-22.9^{\circ}$ & $-125.2^{\circ}$ & $-7.7^{\circ}$ \\
\hline
\end{tabular}

If one were to apply the prescribed parameters to this particular reconstruction, the prescription would have been fulfilled earlier with the event 2007-069 (10 March 2007, marked with a double star in the table), with 5 events in correlation out of 6 above $56 \mathrm{EeV}$.

\section{References}

[1] Pierre Auger Collaboration, Science 318 (2007) 938.

[2] J. Linsley, Phys. Rev. Lett. 10 (1963) 146.

[3] K. Greisen, Phys. Rev. Lett. 16 (1966) 748.

[4] G.T. Zatsepin, V.A. Kuz'min, Sov. Phys. JETP Lett. 4 (1966) 78.

[5] J.L. Puget, F.W. Stecker, J.J. Bredekamp, Astrophys. J. 205 (1976) 638.

[6] L.N. Epele, E. Roulet, Phys. Rev. Lett. 81 (1999) 3295.

[7] J. Abraham et al., Pierre Auger Collaboration, Nucl. Instr. and Meth. A 523 (2004) 50.

[8] V.L. Ginzburg, S.I. Syrovatskii, The Origin of Cosmic Rays, Pergamon Press, Oxford, 1964.

[9] A.M. Hillas, Ann. Rev. Astron. Astrophys. 22 (1984) 425.

[10] M. Ave, Pierre Auger Collaboration, in: Proceedings of the 30th International Cosmic Ray Conference, Mérida, México, 2007. $<$ arXiv:0709.2125>.

[11] M.-P. Véron-Cetty, P. Véron, Astron. Astrophys. 455 (2006) 773, We acknowledge use of the VizieR catalogue access tool, CDS, Strasbourg, France, at $<$ http://vizier.u-strasbg.fr/viz-bin/VizieR $>$.

[12] C. Bonifazi, A. Letessier-Selvon, E.M. Santos, Astropart. Phys. 28 (2008) 523.
[13] C. Bonifazi, Pierre Auger Collaboration, in: Proceedings of the 29th International Cosmic Ray Conference Pune, India, vol. 7, 2005, p. 17.

[14] M. Roth, Pierre Auger Collaboration, in: Proceedings of the 30th International Cosmic Ray Conference, Mérida, México, 2007. $<$ arXiv:0706.2096>.

[15] B. Dawson, Pierre Auger Collaboration, in: Proceedings of the 30th International Cosmic Ray Conference, Mérida, México, 2007. $<$ arXiv:0706.1105>.

[16] D. Allard et al., Pierre Auger Collaboration, in: Proceedings of the 29th International Cosmic Ray Conference, Pune, India, vol. 7, 2005, p. 71. <arXiv:astro-ph/0511104>.

[17] C. Bleve, Pierre Auger Collaboration, in: Proceedings of the 30th International Cosmic Ray Conference, Mérida, México, 2007. $<$ arXiv:0706.1491v1>.

[18] C.B. Finley, S. Westerhoff, Astropart. Phys. 21 (2004) 359.

[19] N. Hayashida et al., Astropart. Phys. 10 (1999) 333.

[20] J.A. Bellido et al., Astropart. Phys. 15 (2001) 167.

[21] N. Hayashida et al., Phys. Rev. Lett. 77 (1996) 1000.

[22] P.G. Tinyakov, I.I. Tkachev, JETP Lett. 74 (2001) 445.

[23] Pierre Auger Collaboration, Astropart. Phys. 27 (2007) 244.

[24] R.U. Abbasi et al., HiRes Collaboration, Astrophys. J. 610 (2004) L73.

[25] R.U. Abbasi et al., HiRes Collaboration, Astrophys. J. 636 (2006) 680.

[26] S. Mollerach, Pierre Auger Collaboration, in: Proceedings of the 30th International Cosmic Ray Conference, Mérida, México, 2007. $<$ arXiv:0706.1749>.

[27] D. Harari, Pierre Auger Collaboration, in: Proceedings of the 30th International Cosmic Ray Conference, Mérida, México, 2007. $<$ arXiv:0706.1715>. 
[28] R.W. Clay, Pierre Auger Collaboration, in: Proceedings of the 28th International Cosmic Ray Conference, Tsukuba, Japan, 2003, p. 421. $<$ arXiv:astro-ph/0308494>.

[29] B. Revenu, Pierre Auger Collaboration, in: Proceedings of the 29th International Cosmic Ray Conference, Pune, India, 2005. <arXiv:astro-ph/0507600>

[30] A. Wald, Ann. Math. Statist. 16 (1945) 117

[31] A. Wald, Sequential Analysis, John Wiley and Sons, New York, 1947.

[32] S.Y. BenZvi, B.M. Connolly, S. Westerhoff, Astrophys. J., submitted for publication. <arXiv:0711.3937>.

[33] P. Sommers, Astropart. Phys. 14 (2001) 271.

[34] P. Billoir, O. Deligny, JCAP 02 (2008) 009.

[35] S.L. Dubovsky, P.G. Tinyakov, I.I. Tkachev, Phys. Rev. Lett. 85 (2000) 1154.

[36] Pierre Auger Collaboration, Discriminating among different scenarios for the correlation of the highest-energy Auger events with nearby sources, in preparation.

[37] W. Saunders et al., Mont. Notice Royal Astron. Soc. 316 (2000) 55.

[38] G.O. Abell, H.G. Corwin, R.P. Olowin, Astrophys. J. Suppl. Ser. 70 (1989) 1.

[39] H. Böhringer et al., Astron. Astrophys. 425 (2004) 367.

[40] H. Böhringer et al., Astrophys. J. Suppl. Ser. 129 (2000) 435.

[41] R. Coziol et al., Astrophys. J. Suppl. Ser. 119 (1998) 239.

[42] F.W. Stecker, Phys. Rev. Lett. 21 (1968) 1016.

[43] D. Harari, S. Mollerach, E. Roulet, JCAP 11 (2006) 012.

[44] R. Engel, Pierre Auger Collaboration, in: Proceedings of the 30th International Cosmic Ray Conference, Mérida, México, 2007. $<$ arXiv:0706.1921>.

[45] T. Stanev, Astrophys. J. 479 (1997) 290.

[46] D. Harari, S. Mollerach, E. Roulet, JHEP 8 (1999) 022.

[47] J.L. Han, in: Supplement: Proceedings of the 2005 Lake Hanas International Pulsar Symposium, Chinese J. Astron. Astrophys. 6 (2006) 211. <arXiv:astro-ph/0603512>

[48] K. Dolag, D. Grasso, V. Springel, I. Tkachev, JCAP 0501 (2005) 009.
[49] G. Sigl, F. Miniati, T.A. Ensslin, Phys. Rev. D 70 (2004) 043007. $<$ arXiv:astro-ph/0401084> .

[50] T. Stanev, High Energy Cosmic Rays, Springer-Verlag, Berlin, 2003.

[51] P. Blasi, R.I. Epstein, A.V. Olinto, Astrophys. J. 533 (2000) L123.

[52] W. Bednarek, Mon. Notice Royal Astron. Soc. 345 (2003) 847.

[53] A. Dar, R. Plaga, Astron. Astrophys. 349 (1999) 259.

[54] V. Berezinsky, M. Kachelriess, A. Vilenkin, Phys. Rev. Lett. 79 (1997) 4302.

[55] V.A. Kuz'min, V.A. Rubakov, Phys. Atom. Nucl. 61 (1998) 1028.

[56] M. Birkel, S. Sarkar, Astropart. Phys. 9 (1998) 297.

[57] Pierre Auger Collaboration <arXiv:0712.1147>.

[58] D. Semikoz, Pierre Auger Collaboration, in: Proceedings of the 30th International Cosmic Ray Conference, Mérida, México, 2007. $<$ arXiv:0706.2960>.

[59] C.T. Hill, Nucl. Phys. B 224 (1983) 469.

[60] D.N. Schramm, C.T. Hill, in: Proceedings of the 18th International Cosmic Ray Conference, Bangalore, India, vol. 2, 1983, p. 393.

[61] For a review see P. Bhattacharjee, G. Sigl, Phys. Rept. 327 (2000) 109.

[62] J. Arons, Astrophys. J. 589 (2003) 871.

[63] E. Boldt, P. Ghosh, Mon. Notice Royal Astron. Soc. 307 (1999) 491

[64] L.A. Anchordoqui, G.E. Romero, J.A. Combi, Phys. Rev. D 60 (1999) 103001

[65] E. Waxman, Astrophys. J. 452 (1995) L1.

[66] M. Vietri, Astrophys. J. 453 (1995) 883.

[67] H. Kang, D. Ryu, T.W. Jones, Astrophys. J. 456 (1996) 422.

[68] H. Kang, J.P. Rachen, P.L. Biermann, Mon. Notice Royal Astron. Soc. 286 (1997) 257

[69] P.L. Biermann, P. Strittmatter, Astrophys. J. 322 (1987) 643.

[70] M. Ostrowski, Astron. Astrophys. 335 (1999) 134.

[71] M. Lyutikov, R. Ouyed, Astropart. Phys. 27 (2007) 473.

[72] J.P. Rachen, P.L. Biermann, Astron. Astrophys. 272 (1993) 161.

[73] F.P. Israel, Astron. Astrophys. Rev. 8 (1998) 237.

[74] G.E. Romero, J.A. Combi, S.E. Perez Bergliaffa, L.A. Anchordoqui, Astropart. Phys. 5 (1996) 279. 